\title{
Seismic response of bridge piers on pile groups for different soil damping models and lumped parameter representations of the foundation*
}

\author{
Francisco González ${ }^{\dagger, a}$, Luis A. Padrón ${ }^{\mathrm{a}}$, Sandro Carbonari ${ }^{\mathrm{b}}$, Michele Moricic ${ }^{\mathrm{c}}$, Juan \\ J. Aznárez ${ }^{\mathrm{a}}$, Francesca Dezi ${ }^{\mathrm{d}}$, and Graziano Leonic ${ }^{\mathrm{c}}$ \\ a SIANI, Universidad de Las Palmas de Gran Canaria, Spain \\ ${ }^{\mathrm{b}}$ DICEA, Università Politecnica delle Marche, Ancona, Italy \\ ' $S A A D$, University of Camerino, Ascoli Piceno, Italy \\ ${ }^{\mathrm{d}}$ DESD, University of San Marino, Republic of San Marino
}

\begin{abstract}
This paper presents a wide parametric study aimed at elucidating the influence, on the computed seismic response of bridge piers, of two related aspects of the model: i) the adoption of the classical hysteretic or the causal Biot's damping models for the soil, and ii) the use of two different lumped parameter models of different complexity and accuracy to approximate the impedances of the pile foundation. A total of 2072 cases, including different superstructures, pile foundations, soil deposits and seismic input signals, are studied. The results are presented so that the influence of the different parameters involved in the analysis can be assessed. From an engineering point of view, both lumped parameter models provide, in general, sufficiently low errors. The choice of the most adequate model for each case will depend not only on the configuration of the structure and the soil-foundation system, but also on the assumed soil damping model, whose influence on the computed seismic responses is relevant in many cases. The non-physical behaviour provided by the classical hysteretic damping model for the soil at zero frequency generates issues in the process of fitting the impedance functions. It is also found that larger deck displacements are predicted by Biot's model due to the higher damping at low frequencies provided by the classical hysteretic damping model.
\end{abstract}

Keywords: soil-structure interaction, hysteretic damping model, Biot's damping model, lumped parameter model, bridge seismic response, pile foundations.

${ }^{*}$ This is the peer reviewed version of the following article: F. González, L. A. Padrón, S. Carbonari, M. Morici, J. J. Aznárez, F. Dezi, G. Leoni, Seismic response of bridge piers on pile groups for different soil damping models and lumped parameter representations of the foundation, Earthquake Engineering \& Structural Dynamics, 48(3):306327, 2019, which has been published in final form at https://doi.org/10.1002/eqe.3137. This article may be used for non-commercial purposes in accordance with Wiley Terms and Conditions for Self-Archiving.

${ }^{\dagger}$ Corresponding author: Francisco González, Instituto Universitario de Sistemas Inteligentes y Aplicaciones Numéricas en Ingeniería, Universidad de Las Palmas de Gran Canaria, Edificio Central del Parque Científico y Tecnológico del Campus Universitario de Tafira, 35017 Las Palmas de Gran Canaria, Spain.

Email: francisco.gonzalez@ulpgc.es 


\section{Introduction}

Dynamic Soil-Structure Interaction (SSI) is known to be a factor that can affect significantly the seismic response of bridges $[1,2,3,4]$. When this is the case, the structural model used in the analyses should be able to take into account, as rigorously as possible, the complex dynamic response of foundations such as the pile group. Such response can be efficiently characterized in the frequency domain when the soil-foundation system is assumed to remain in the linear-elastic range. However, if non-linear phenomena need to be considered in the bridge superstructure, the analyses should be carried out in the time-domain. In this case, if the response of the soil-foundation system is assumed to remain in linear-elastic range, stiffness and damping functions obtained in the frequency domain to characterize the response of the foundations can still be incorporated into the analysis, for instance, by approximating them by pertinent Lumped Parameter Models (LPMs) that can subsequently be used to represent the response of the soil-foundation system in a substructuring scheme $[2,4,5,6,7]$.

Such LPMs are simple systems, with relatively few degrees of freedom, tuned to approximate the dynamic response of a different system (for instance, a foundation) both in the frequency and the time domains. Their application to SSI problems gained momentum with the seminal papers by Wolf $[8,9]$ proposing the consistent approach, although other simple schemes had already been previously used $[5,10,11,12]$. Thus, there exist now different possible LPMs, with different levels of complexity and accuracy, that can be adopted. However, it is still unclear whether it is justified to use complex LPMs when the aim of the study is analysing the response of the superstructure, and taking into account the simplifying assumptions and the uncertainty associated to all elements in the model built to represent the whole system.

Part of such uncertainty resides in the model adopted to represent the behaviour of the soil. The absence of non-linear response in the foundation is a common simplifying hypothesis, justified by the fact that, in general, foundations must be designed and detailed to avoid substantial permanent deformations [13] and because of the complexity of dealing with the frequency-dependent non-linear nature of soil. Another assumption is related to the model of energy dissipation in the soil. In this regard, classical frequency-independent material damping (labelled as "hysteretic damping" by Bishop [14] in 1955) is the most common choice when the analysis is performed in the frequency domain [15], partly due to its simplicity. This is the case even though it is well known that it gives rise to non-causal and irrealizable structural models. Besides, the adoption of this damping model leads to frequency response functions with non-zero imaginary parts at zero frequency, which lacks of physical meaning. However, there exist alternative damping models that also show a frequencyindependent (or quasi-independent) hysteretic damping but are, at the same time, causal, though rarely used in structural analysis. For such damping models to be causal, it is condition both necessary and sufficient to satisfy the equations of Kramers-Kronig [16], which relates the real and the imaginary parts of the mathematical model. The first hysteretic damping model both causal and with a damping rate almost frequency-independent was proposed by Biot [17] in 1958, with real and imaginary parts both variable with frequency. Later, Makris [18] proposed a model whose initial premise was the invariability of the damping rate with frequency, giving rise to a function whose real part is bounded for all frequencies except for $\omega=0$, point at which it tends to $-\infty$. For this reason, the use of Biot's model is preferred in this research, even if both are causal models suitable for frequency-domain SSI analysis, and both provide very close results for low and medium frequencies. In this respect, and despite the time since its publication, the magnitude of the influence of the use of Biot's or the classical hysteretic damping model on the seismic response of the superstructure is still unclear

Thus, the aim of this paper is to quantify the influence, on the computed seismic response of bridge piers on pile groups, of two closely related aspects: i) the adoption of the classical non-causal hysteretic damping model for the soil, or the causal Biot's damping model; and ii) the implementation of a relatively complex consistent LPM based on a rational approximation, or a more simplified 
LPM, to represent the behaviour of the foundation. As will be shown later, both aspects are studied simultaneously because the choice of soil damping can significantly influence the ability of the LPMs to capture the impedances of the pile group foundations. To achive this goal, a wide parametric study involving different soil profiles and superstructures is carried out in order to be able to draw general conclusions. The analysis is carried out into a linear-elastic framework so that the conclusions of the study provide an informed starting point for the study of the more involved non-linear case.

\section{Problem description}

\subsection{Superstructures and foundation layouts}

The study presented in this paper focuses on the seismic response of single bridge piers founded on pile foundations, using a simplified model that takes SSI into account, and that may be representative of the transverse response of bridges with simply supported decks and of multispan continuous decks not connected at the abutments in the transverse direction. In the case of bridges with a dual load path mechanism (i.e. for bridges with deck connected to the abutments in the transverse direction), the model may be representative of the transverse seismic behaviour of inner piers where effects of the boundary conditions are negligible. Figure 1 represents the model of the soil-foundation-pier system (see e.g. Carbonari et al. [19]). The pile cap and the bent cap are assumed to be rigid, while the elastic and damping properties of the pier are modelled through a lumped rotational spring $\left(K_{\phi}\right)$ and dashpot $\left(C_{\phi}\right)$ at the base of the pier. The bridge is assumed to be founded on pile groups. SSI analyses are performed through the sub-structure [20] approach and the compliance of the soil-foundation system is represented by the frequency-dependent impedance matrix of the soil-foundation system. The pier is subjected to the Foundation Input Motion (FIM) [21], namely the motion experienced by the foundation as a consequence of the seismic waves propagating into the soil. This can be obtained starting from the free-field motion and the kinematic interaction factors of the soil-foundation system, resulting from the analysis of the soil-foundation sub-domain subjected to propagating harmonic shear waves in the soil. In Figure 1, $m_{d}$ and $m_{c}$ are the masses of bridge deck portion belonging to the generic pier and of the pier bent cap, respectively. Furthermore, $m_{p}$ and $m_{f}$ are the masses of the pier and the pile cap, and $I_{d}, I_{c}$ and $I_{f}$ are the mass moments of inertia of the deck and the bent cap, and of the pile cap, respectively. Finally, $h_{d}, h_{c}, h_{p}$ and $h_{f}$ are dimensions necessary to define the heights of the mass centroids of the deck, the bent cap and the pile cap, respectively. By considering the in-plane response of the soil-foundation-pier system, the whole problem is described as a three degree-of-freedom system, with $u_{F}$ and $\varphi_{F}$ being the translation and rotation of the foundation, and $\varphi_{S}$ the relative rotation of the pier with respect to the foundation. $u_{g}$ and $\varphi_{g}$ are the translational and rotational FIM. It is worth noting that, by assuming pile layouts characterised by two symmetry axes and by referring the impedance matrix of the soil-foundation systems to the centroids of the pile configuration at the level of the pile head, the vertical degree-of-freedom is uncoupled from the horizontal and rotational ones and is not included in the formulation. Furthermore, significant components of the soil-foundation impedance matrix are the horizontal, rotational and coupled roto-translational terms, represented in the Compliant-Base (CB) model of the pier in Figure 1 through linear frequency dependent visco-elastic Kelvin-Voigt's models at the foundation-superstructure interface (centroid of piles configurations at the level of the pile head).

Four realistic superstructures, characterised by fundamental periods $T_{n}=0.2 \mathrm{~s}, T_{n}=0.5 \mathrm{~s}$, $T_{n}=1.0 \mathrm{~s}$ and $T_{n}=1.5 \mathrm{~s}$, are considered in this study (see Table 1). Mass and geometric properties comply with steel-concrete composite continuous bridge decks with span length of $25 \mathrm{~m}$. A forcebased approach is adopted to design the fixed base piers on both soil deposits, obtaining longitudinal reinforcement ratios ranging between $1.3-3.0 \%$. Piers are founded on six different pile foundations, constituted by $2 \times 2$ and $3 \times 3$ vertical piles groups with diameter $d=1.0 \mathrm{~m}$, pile length $L=20.0 \mathrm{~m}$, 

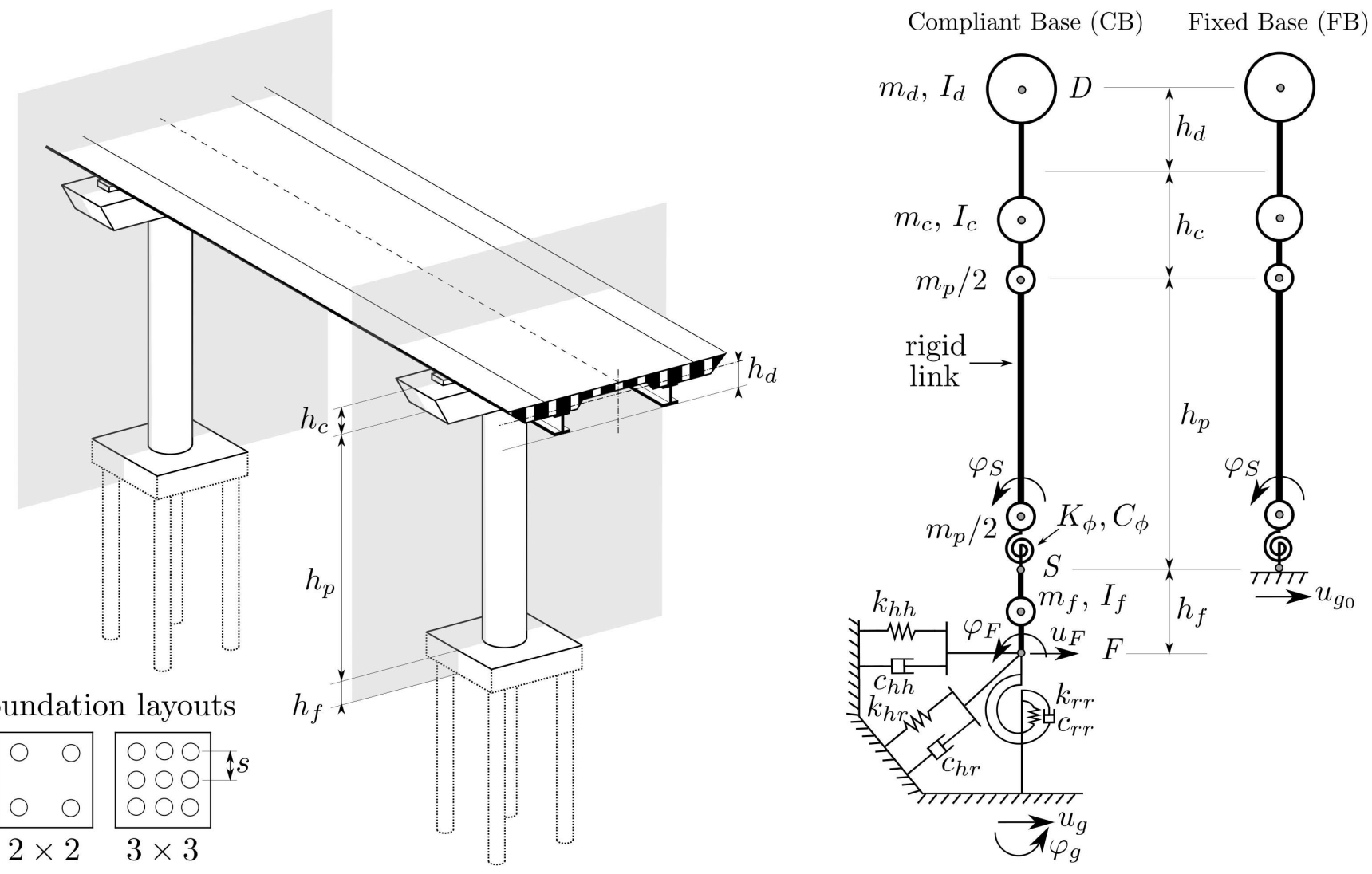

Figure 1: Soil-foundation-superstructure system (left), and substructure models of piers cross sections (right) [19].

and three different pile-to-pile spacings $s(3,5$ and $7 \mathrm{~m})$. Foundations are selected to balance the need of parametrically addressing the effects of the foundation deformability on the superstructure response with that of considering realistic scenarios. Overall, they comply with stress resultants at the pier bases in the $80 \%$ of cases, being the $2 \times 2$ layout with spacing $3 \mathrm{~m}$ and $3 \times 3$ layout with spacing $7 \mathrm{~m}$ border line configurations. The pile cap mass and inertial properties, which depend on the foundation layouts, are summarized in Table 2.

Piles Young's modulus and density are $E_{p}=30.0 \mathrm{GPa}$ and $\rho_{p}=2.5 \mathrm{t} / \mathrm{m}^{3}$, respectively. The pier elastic flexural spring $\left(K_{\phi}\right)$ is calibrated to reproduce the fundamental period of the system, while the damping coefficient $\left(C_{\phi}\right)$ is calibrated to obtain a $5 \%$ viscous damping ratio at the fundamental period of the pier. Fixed-Base (FB) models will be also considered (Figure 1), whose results will be used to address and discuss the significance of the SSI effects.

\subsection{Soil properties and seismic actions}

Foundations are assumed to be embedded in two different soil deposits, representative of a soil type D (loose-to-medium cohesionless soil or predominantly soft-to-firm cohesive soil) and of a soil type $\mathrm{C}$ (dense or medium-dense sand, gravel or stiff clay), according to Eurocode 8 [22]. With reference to the softer soil deposit, a soil density $\rho_{s}=1.56 \mathrm{t} / \mathrm{m}^{3}$, shear wave velocity $c_{s}=117.1$ $\mathrm{m} / \mathrm{s}$, soil Poisson's ratio $\nu_{s}=0.4$ and soil damping coefficient $\xi_{s}=5 \%$ are assumed, while for the stiffer deposit, representative of a ground type C, a soil density $\rho_{s}=1.67 \mathrm{t} / \mathrm{m}^{3}$, shear wave velocity $c_{s}=253.5 \mathrm{~m} / \mathrm{s}$, Poisson's ratio $\nu_{s}=0.4$ and soil damping coefficient $\xi_{s}=5 \%$ are considered.

The seismic action is constituted by a set of 7 real accelerograms for each soil deposit, selected from the European and Italian strong motion databases [23, 24]. The selection criterion is based on both the Magnitude $\left(\mathrm{M}_{\mathrm{w}}>5\right)$ and the site classification (records registered on soil type $\mathrm{C}$ and 


\begin{tabular}{l|cccc} 
Superstructure & $\mathbf{1}$ & $\mathbf{2}$ & $\mathbf{3}$ & $\mathbf{4}$ \\
\hline$T_{n}[\mathrm{~s}]$ & 0.2 & 0.5 & 1.0 & 1.5 \\
$f_{n}[\mathrm{~Hz}]$ & 5.0 & 2.0 & 1.0 & $0 . \overline{6}$ \\
\hline$m_{d}[\mathrm{t}]$ & 305.8 & 305.8 & 305.8 & 305.8 \\
$I_{d}\left[\mathrm{t} \mathrm{m}{ }^{2}\right]$ & 2466.6 & 2466.6 & 2466.6 & 2466.6 \\
$h_{d}[\mathrm{~m}]$ & 0.59 & 0.59 & 0.59 & 0.59 \\
$m_{c}[\mathrm{t}]$ & 88.3 & 88.3 & 88.3 & 88.3 \\
$I_{c}\left[\mathrm{t} \mathrm{m}{ }^{2}\right]$ & 426.6 & 426.6 & 426.6 & 426.6 \\
$h_{c}[\mathrm{~m}]$ & 1.80 & 1.80 & 1.80 & 1.80 \\
$m_{p}[\mathrm{t}]$ & 57.6 & 121.1 & 196.0 & 253.6 \\
$h_{p}[\mathrm{~m}]$ & 5.0 & 10.5 & 17.0 & 22.0 \\
$K_{\phi}[\mathrm{MN} / \mathrm{rad}]$ & 23067.6 & 10984.6 & 6784.6 & 5242.6 \\
\hline Real period $[\mathrm{s}]$ & 0.2001 & 0.5082 & 1.0076 & 1.4841
\end{tabular}

Table 1: Superstructures parameters.

\begin{tabular}{l|ccc|ccc} 
& \multicolumn{3}{|c|}{$2 \times 2$} & \multicolumn{3}{|c}{$3 \times 3$} \\
& $s=3[\mathrm{~m}]$ & $s=5[\mathrm{~m}]$ & $s=7[\mathrm{~m}]$ & $s=3[\mathrm{~m}]$ & $s=5[\mathrm{~m}]$ & $s=7[\mathrm{~m}]$ \\
\hline$h_{f}[\mathrm{~m}]$ & 1.5 & 2.0 & 2.5 & 2.0 & 2.5 & 3.0 \\
$m_{f}[\mathrm{t}]$ & 93.75 & 245.00 & 506.25 & 320.00 & 900.00 & 1920.00 \\
$I_{f}\left[\mathrm{t} \mathrm{m}^{2}\right]$ & 212.89 & 1082.08 & 3680.86 & 1813.33 & 11268.75 & 42400.00
\end{tabular}

Table 2: Pile cap properties depending on foundation layout for CB model.

D); the limited number of eligible records for selection required the inclusion of mainshock and aftershocks of the same event. The selected records are reported in Tables 3 and 4 for deposits D and C, respectively. For each superstructure, accelerograms are scaled in order to obtain the matching of the pseudo spectral acceleration of each accelerogram with the response spectrum proposed in the standard [22] at the fundamental periods of the FB systems, as shown in Figure 2 in which the mean response spectrum of the scaled set of accelerograms are reported for each superstructure on both soil deposits. It worth mentioning that low scale factors are used (overall between 0.25 and 3.0), reducing bias in the structural response caused by the ground motion selection $[25,26,27]$.

\section{Methodology}

\subsection{Equations of motion}

By assuming a linear elastic behaviour for the pier and neglecting second-order effects (i.e. making the hypothesis of small displacements), the equations of motion of the CB system shown in Figure 1 may be conveniently written in the frequency domain as

$$
\begin{array}{r}
\left.\left[\begin{array}{ccc}
K_{\phi} & 0 & 0 \\
0 & k_{h h}(\omega) & k_{h r}(\omega) \\
0 & k_{r h}(\omega) & k_{r r}(\omega)
\end{array}\right]-\omega^{2}\left[\begin{array}{ccc}
m_{11} & m_{12} & m_{13} \\
m_{12} & m_{22} & m_{23} \\
m_{13} & m_{23} & m_{33}
\end{array}\right]+\mathrm{i} \omega\left[\begin{array}{ccc}
C_{\phi} & 0 & 0 \\
0 & c_{h h}(\omega) & c_{h r}(\omega) \\
0 & c_{r h}(\omega) & c_{r r}(\omega)
\end{array}\right]\right\}\left[\begin{array}{c}
\Phi_{S} \\
U_{F} \\
\Phi_{F}
\end{array}\right]= \\
=\omega^{2}\left[\begin{array}{ccc}
m_{11} & m_{12} & m_{13} \\
m_{12} & m_{22} & m_{23} \\
m_{13} & m_{23} & m_{33}
\end{array}\right]\left[\begin{array}{ll}
0 & 0 \\
1 & 0 \\
0 & 1
\end{array}\right]\left[\begin{array}{l}
U_{g} \\
\Phi_{g}
\end{array}\right]
\end{array}
$$




\begin{tabular}{|c|c|c|c|c|c|c|}
\hline Database & $\begin{array}{c}\text { Earthquake } \\
\text { WC-EC }\end{array}$ & Station ID & $\begin{array}{c}\text { Date } \\
{[\mathrm{dd} / \mathrm{mm} / \mathrm{yy}]}\end{array}$ & $\begin{array}{c}\Delta \\
{[\mathrm{km}]}\end{array}$ & $\begin{array}{c}\text { Magnitude } \\
{\left[\mathrm{M}_{\mathrm{w}}\right]}\end{array}$ & $\begin{array}{c}P G A \\
{\left[\mathbf{m} / \mathbf{s}^{2}\right.}\end{array}$ \\
\hline ITACA & $\begin{array}{c}\text { Val Nerina } \\
138-036\end{array}$ & BVG & 19/09/1979 & 38.0 & 5.8 & $\begin{array}{l}0.222 \\
y \text {-dir }\end{array}$ \\
\hline ITACA & $\begin{array}{c}\text { Umbria-Marche } 1^{\text {st }} \text { shock } \\
\quad 363-099\end{array}$ & BVG & 26/09/1997 & 24.9 & 5.7 & $\begin{array}{l}0.372 \\
x-\operatorname{dir}\end{array}$ \\
\hline ITACA & $\begin{array}{c}\text { Umbria-Marche } 2^{\text {nd }} \text { shock } \\
\qquad 394-100\end{array}$ & RTI & 26/09/1997 & 65.0 & 6.0 & $\begin{array}{l}0.184 \\
y \text {-dir }\end{array}$ \\
\hline ITACA & $\begin{array}{c}\text { App. Umbro-Marchigiano } \\
429-111\end{array}$ & BVG & 06/10/1997 & 21.9 & 5.4 & $\begin{array}{l}0.349 \\
x-\operatorname{dir}\end{array}$ \\
\hline ITACA & $\begin{array}{c}\text { Umbria-Marche } 3^{\text {rd }} \text { shock } \\
\qquad 482-118\end{array}$ & BVG & $14 / 10 / 1997$ & 23.8 & 5.6 & $\begin{array}{l}0.359 \\
y \text {-dir }\end{array}$ \\
\hline ESD & $\begin{array}{c}\text { Izmit (aftershock) } \\
6947-2154\end{array}$ & 3270 & 31/08/1999 & 39 & 5.1 & $\begin{array}{l}0.358 \\
y \text {-dir }\end{array}$ \\
\hline ESD & $\begin{array}{c}\text { Izmit (aftershock) } \\
6967-0473\end{array}$ & 767 & 13/09/1999 & 120 & 5.8 & $\begin{array}{l}0.391 \\
x-\text { dir }\end{array}$ \\
\hline
\end{tabular}

Table 3: Selected records for ground type D (WC: Waveform Code and EC: Earthquake Code) [23, 24].

where mass matrix coefficients are:

$$
\begin{aligned}
m_{11}= & \left(h_{p}+h_{c}+h_{d}\right)^{2} m_{d}+I_{d}+\left(h_{p}+\frac{h_{c}}{2}\right)^{2} m_{c}+I_{c}+h_{p}^{2} \frac{m_{p}}{2} \\
m_{12}= & \left(h_{p}+h_{c}+h_{d}\right) m_{d}+\left(h_{p}+\frac{h_{c}}{2}\right) m_{c}+h_{p} \frac{m_{p}}{2} \\
m_{13}= & \left(h_{p}+h_{c}+h_{d}\right)\left(h_{f}+h_{p}+h_{c}+h_{d}\right) m_{d}+I_{d}+\left(h_{p}+\frac{h_{c}}{2}\right)\left(h_{f}+h_{p}+\frac{h_{c}}{2}\right) m_{c}+I_{c}+ \\
& +h_{p}\left(h_{f}+h_{p}\right) \frac{m_{p}}{2} \\
m_{22}= & m_{d}+m_{c}+m_{p}+m_{f} \\
m_{23}= & \left(h_{f}+h_{p}+h_{c}+h_{d}\right) m_{d}+\left(h_{f}+h_{p}+\frac{h_{c}}{2}\right) m_{c}+\left(h_{f}+h_{p}\right) \frac{m_{p}}{2}+h_{f} \frac{m_{p}}{2}+\frac{h_{f}}{2} m_{f} \\
m_{33}= & \left(h_{f}+h_{p}+h_{c}+h_{d}\right)^{2} m_{d}+I_{d}+\left(h_{f}+h_{p}+\frac{h_{c}}{2}\right)^{2} m_{c}+I_{c}+\left(h_{f}+h_{p}\right)^{2} \frac{m_{p}}{2}+h_{f}^{2} \frac{m_{p}}{2}+ \\
& +\left(\frac{h_{f}}{2}\right)^{2} m_{f}+I_{f}
\end{aligned}
$$

being $\omega$ the frequency of excitation and "i" the imaginary unit. Stiffness and damping coefficients shown in Equation (1) are obtained from the complex-valued frequency-dependent impedance functions $Z_{h h}(\omega)=k_{h h}(\omega)+\mathrm{i} \omega c_{h h}(\omega), Z_{r r}(\omega)=k_{r r}(\omega)+\mathrm{i} \omega c_{r r}(\omega)$ and $Z_{h r}(\omega)=k_{h r}(\omega)+\mathrm{i} \omega c_{h r}(\omega)$, representing the stiffness and damping of the foundation in the horizontal, rocking and cross-coupled horizontal-rocking vibration modes respectively.

In Equation(1), $\Phi_{S}, U_{F}$ and $\Phi_{F}$ are the Fourier Transform of the superstructure and foundation generalised relative displacements $\left(\varphi_{S}, u_{F}\right.$ and $\varphi_{F}$, respectively), while $U_{g}$ and $\Phi_{g}$ are the Fourier Transform of the generalised displacements constituting the FIM derived from the kinematic interaction analysis of the pile foundation $\left(u_{g}\right.$ and $\varphi_{g}$, respectively). The three equations of motion describe the moment equilibrium of the pier, the horizontal force and moment equilibrium of the 


\begin{tabular}{|c|c|c|c|c|c|c|}
\hline Database & $\begin{array}{c}\text { Earthquake } \\
\text { WC-EC }\end{array}$ & Station ID & $\begin{array}{c}\text { Date } \\
{[\mathrm{dd} / \mathrm{mm} / \mathrm{yy}]}\end{array}$ & $\begin{array}{c}\Delta \\
{[\mathbf{k m}]}\end{array}$ & $\begin{array}{c}\text { Magnitude } \\
{\left[\mathrm{M}_{\mathrm{w}}\right]}\end{array}$ & $\begin{array}{c}P G A \\
{\left[\mathbf{m} / \mathbf{s}^{2}\right]}\end{array}$ \\
\hline ESD & $\begin{array}{c}\text { Friuli (aftershock) } \\
133-63\end{array}$ & 33 & 15/09/1976 & 9 & 6.0 & $\begin{array}{l}0.932 \\
y \text {-dir }\end{array}$ \\
\hline ESD & $\begin{array}{c}\text { Campano Lucano } \\
299-146\end{array}$ & 105 & $23 / 11 / 1980$ & 52 & 6.9 & $\begin{array}{l}0.471 \\
x-\text { dir }\end{array}$ \\
\hline ESD & $\begin{array}{c}\text { Manjil } \\
475-230\end{array}$ & 184 & 20/06/1990 & 91 & 7.4 & $\begin{array}{l}1.295 \\
x-\text { dir }\end{array}$ \\
\hline ESD & $\begin{array}{c}\text { Racha (aftershock) } \\
529-248\end{array}$ & 199 & $15 / 06 / 1991$ & 72 & 6.0 & $\begin{array}{l}0.169 \\
x-\text { dir }\end{array}$ \\
\hline ESD & $\begin{array}{c}\text { Izmit } \\
1251-472\end{array}$ & 773 & 17/08/1999 & 92 & 7.6 & $\begin{array}{l}0.911 \\
x-\text { dir }\end{array}$ \\
\hline ESD & $\begin{array}{c}\text { Ishakli } \\
7097-2295\end{array}$ & 856 & 03/02/2002 & 66 & 6.5 & $\begin{array}{l}1.106 \\
x-\text { dir }\end{array}$ \\
\hline ESD & $\begin{array}{c}\text { Ishakli (aftershock) } \\
7104-2296\end{array}$ & 856 & $03 / 02 / 2002$ & 35 & 5.8 & $\begin{array}{l}0.507 \\
y \text {-dir }\end{array}$ \\
\hline
\end{tabular}

Table 4: Selected records for ground type C (WC: Waveform Code and EC: Earthquake Code) [23].

superstructure-foundation system, respectively. On the other hand, when obtaining the FB response of the structure by solving the corresponding one degree-of-freedom system, no kinematic interaction is considered (i.e., the input signal coincides with the scaled horizontal free-field ground motion $\left.\left(u_{g_{0}}\right)\right)$.

These equations of motion constitute an algebraic system in the frequency domain and the solution can be easily derived by inverting the dynamic stiffness matrix of the system [15]. Once the solution is derived in the frequency domain, the corresponding quantities in the time domain (i.e. time histories of displacements and rotations) are obtained operating the Inverse Fourier Transform.

However, professional engineers are less familiar with frequency domain analyses than time domain analyses. Furthermore, the latter are mandatory if the non-linear superstructure response is taken into account. In this case, Newmark's linear acceleration time-stepping approach can be adopted to obtain the problem solution. The problem formulation in the time domain requires a suitable strategy to account for the frequency-dependent behaviour of the soil-foundation system. Usually, LPMs having impedances that approximate those of the soil-foundation system in a selected frequency range of interest are used [28]. The accuracy of an LPM in reproducing the dynamic behaviour of the soil-foundation system depends on its configuration and degrees of freedom. Use of LPMs leads to some loss of precision, but this is compensated by the advantage of using commercial structural analysis software in performing non-linear analyses. In this paper, the dynamic problem (Figure 1) is both solved in the frequency domain and time domain, by adopting two different LPMs, as described in the following sections, to approximate the soil-foundation system behaviour. From a general point of view, Equation (1) can be written in the time domain according to 


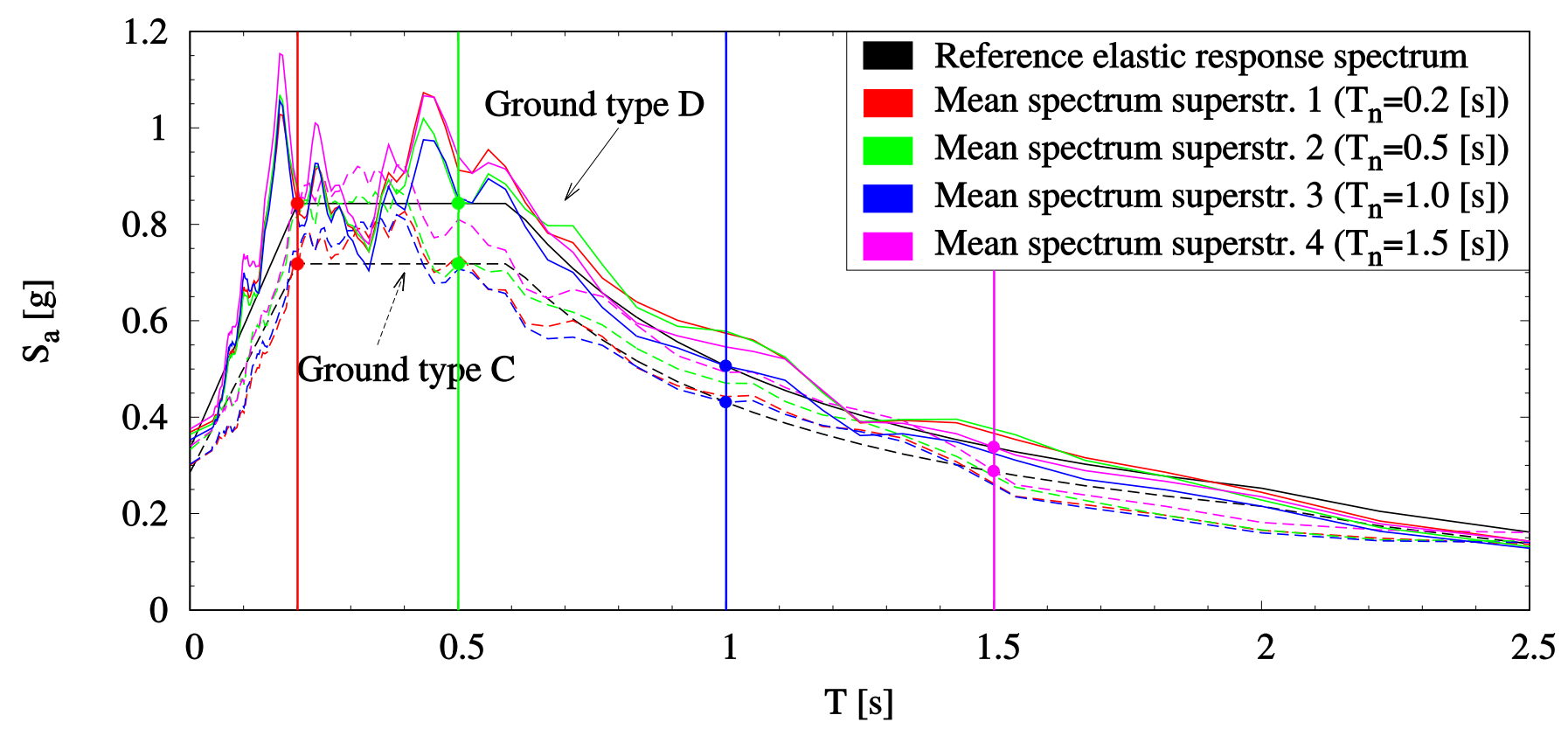

Figure 2: Mean response spectrum of the resulting sets of accelerograms scaled for each superstructure and ground type (Ground type D in continuous line and Ground type C in dashed line).

$$
\begin{gathered}
{\left[\begin{array}{cccc}
m_{11} & m_{12} & m_{13} & \mathbf{0} \\
m_{12} & m_{22}+\bar{m}_{h h} & m_{23}+\bar{m}_{h r} & \mathbf{0} \\
m_{13} & m_{23}+\bar{m}_{r h} & m_{33}+\bar{m}_{r r} & \mathbf{0} \\
\mathbf{0} & \mathbf{0} & \mathbf{0} & \overline{\mathbf{M}}_{l l}
\end{array}\right]\left[\begin{array}{c}
\ddot{\varphi}_{S} \\
\ddot{u}_{F} \\
\ddot{\varphi}_{F} \\
\ddot{\mathbf{u}}_{l}
\end{array}\right]+\left[\begin{array}{cccc}
C_{\phi} & 0 & 0 & \mathbf{0} \\
0 & \bar{c}_{h h} & \bar{c}_{h r} & \overline{\mathbf{c}}_{h l} \\
0 & \bar{c}_{r h} & \bar{c}_{r r} & \overline{\mathbf{c}}_{r l} \\
\mathbf{0} & \overline{\mathbf{c}}_{l h} & \overline{\mathbf{c}}_{l r} & \overline{\mathbf{C}}_{l l}
\end{array}\right]\left[\begin{array}{c}
\dot{\varphi}_{S} \\
\dot{u}_{F} \\
\dot{\varphi}_{F} \\
\dot{\mathbf{u}}_{l}
\end{array}\right]+} \\
+ \\
+\left[\begin{array}{ccccc}
K_{\phi} & 0 & 0 & \mathbf{0} \\
0 & \bar{k}_{h h} & \bar{k}_{h r} & \overline{\mathbf{k}}_{h l} \\
0 & \bar{k}_{r h} & \bar{k}_{r r} & \overline{\mathbf{k}}_{r l} \\
\mathbf{0} & \overline{\mathbf{k}}_{l h} & \overline{\mathbf{k}}_{l r} & \overline{\mathbf{K}}_{l l}
\end{array}\right]\left[\begin{array}{c}
\varphi_{S} \\
u_{F} \\
\varphi_{F} \\
\mathbf{u}_{l}
\end{array}\right]=-\left[\begin{array}{cccc}
m_{11} & m_{12} & m_{13} & \mathbf{0} \\
m_{12} & m_{22} & m_{23} & \mathbf{0} \\
m_{13} & m_{23} & m_{33} & \mathbf{0} \\
\mathbf{0} & \mathbf{0} & \mathbf{0} & \mathbf{0}
\end{array}\right]\left[\begin{array}{ll}
0 & 0 \\
1 & 0 \\
0 & 1 \\
\mathbf{0} & \mathbf{0}
\end{array}\right]\left[\begin{array}{c}
\ddot{u}_{g} \\
\ddot{\varphi}_{g}
\end{array}\right]
\end{gathered}
$$

where the symbol $\bar{\square}$ denotes the frequency independent stiffness, damping and mass parameters of the LPM. Depending on the LPM complexity, hidden degrees of freedom $(l)$ may be included in the equilibrium equations.

\subsection{Modelling and computation of the dynamic soil-pile response}

Impedance functions and kinematic interaction factors of piles foundations used in this work are computed through a frequency-domain boundary element - finite element (BEM-FEM) coupled model previously developed by Padrón et al. [29]. In this type of models, the dissipation of energy in the soil medium is usually modelled through frequency-independent hysteretic damping of the type

$$
\mathscr{E}=\operatorname{Re}[\mathscr{E}](1+\mathrm{i} 2 \xi)
$$

where $\xi$ is the damping coefficient and $\mathscr{E}$ is a material property (shear or Young's modulus, for example). The use of this model is generally justified by the independence between the excitation frequency and the amount of energy dissipated in each cycle for some materials [30, 31], and it is 
massively used due to its convenience in harmonic analysis. However, its use in the analysis of nonstationary (transient) vibrations, either through Fourier analysis or directly in the time domain, is problematic for various reasons [32]. The first feature of this model that can be seen as incongruous is the non-zero imaginary component of the response in $\omega=0$. Further analyses show that its use leads to non-causal models, which implies that a material that responds to a law of the type of the Equation (4) is physically impossible, i.e. in no case can this model respond to reality. Hereafter, this classical hysteretic damping model is labelled as "hysteretic".

The first hysteretic model, both causal and with a damping rate almost independent of frequency, was proposed by Biot in 1958 [17]:

$$
\mathscr{E}(\omega)=\operatorname{Re}[\mathscr{E}]\left(1+\frac{2}{\pi} \xi \ln \sqrt{1+\left(\frac{\omega}{\epsilon_{b}}\right)^{2}}+\mathrm{i} \frac{2}{\pi} \xi \operatorname{atan}\left(\frac{\omega}{\epsilon_{b}}\right)\right)
$$

where both real and imaginary parts vary with frequency but, at $\omega=0$, imaginary part vanishes $(\mathscr{E}(\omega=0) \rightarrow \operatorname{Re}[\mathscr{E}])$. Here, $\epsilon_{b}$ is a real and positive number with the same units than $\omega$, and can be seen as a reference frequency that defines the function variation and for which the material property acquires the value $\mathscr{E}\left(\omega=\epsilon_{b}\right)=\operatorname{Re}[\mathscr{E}](1+0.22 \xi+\mathrm{i} \xi / 2)$. The parameter $\epsilon_{b}$ controls how wide is the detachment to the response computed with the classical hysteretic damping model (Equation (4)). For impedance functions, the effect is produced mainly at lower frequency range for damping coefficients and at higher frequencies for spring coefficient (see for instance Figure 4). In this work, a value of $\epsilon_{b}=0.15 c_{s} /(2 \pi d) \mathrm{Hz}$ is chosen, which derives in two cases depending on the soil type, $\epsilon_{b}=2.80$ $\mathrm{Hz}$ for $\mathrm{D}$ case and $\epsilon_{b}=6.05 \mathrm{~Hz}$ for $\mathrm{C}$.

\subsection{Lumped parameter models}

The two alternative LPMs used in this study to represent the dynamic behaviour of the pile foundations when computing the seismic response of superstructures in the time domain are presented in this section.

On the one hand, a consistent LPM, as described for instance by Wolf [8, 9] or Andersen [33, 34] is used (Figure 3(a)). In this case, each component of the different impedance functions $\left(Z_{h h}\right.$, $Z_{h r}$ and $Z_{r r}$ ) is fitted independently by a rational approximation, or rational filter, consisting of a constant/linear term and $N$ second order terms, leading to approximated impedance functions $\hat{Z}_{h h}, \hat{Z}_{h r}$ and $\hat{Z}_{r r}$. Once the numerator coefficients and poles of the rational filter are estimated, an equivalent mechanical system with few degrees of freedom is established. The different parameters $\left(k_{1 n}, k_{2 n}, k_{3 n}, c_{1 n}, c_{2 n}, c_{3 n}\right.$ and $m_{n}$ as illustrated in Figure 3(a)) that define such a simple system are then computed through an equilibrium formulation between each hidden degree of freedom and the master node. The arising system of equations representing the behaviour of the $\mathrm{CB}$ system is assembled and added to the equations of motion according to Equation (3).

An alternative simplified LPM, based on the work by Carbonari, Dezi and Leoni [36], is also evaluated (Figure 3(b)). This is constituted by a two degree-of-freedom LPM characterized by a translational mass $m_{h}$ and a mass moment of inertia $I_{r}$ at the foundation-structure interface, connected to the soil through translational and rotational pairs of constant springs and dashpots $k_{h}, c_{h}, k_{r}$ and $c_{r}$. Furthermore, masses are connected to an additional eccentric translational mass

$m_{t}$, spring $k_{t}$ and dashpot $c_{t}$ through rigid massless links of lengths $h_{3}$, and $h_{2}$, respectively. The dynamic impedance matrix arising from this model can be expressed as: 
a) Consistent

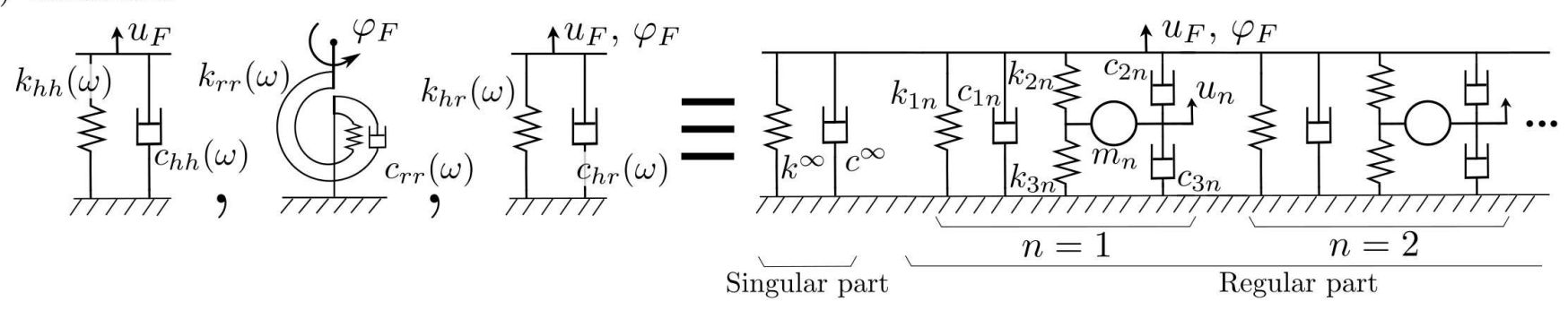

b) Simplified
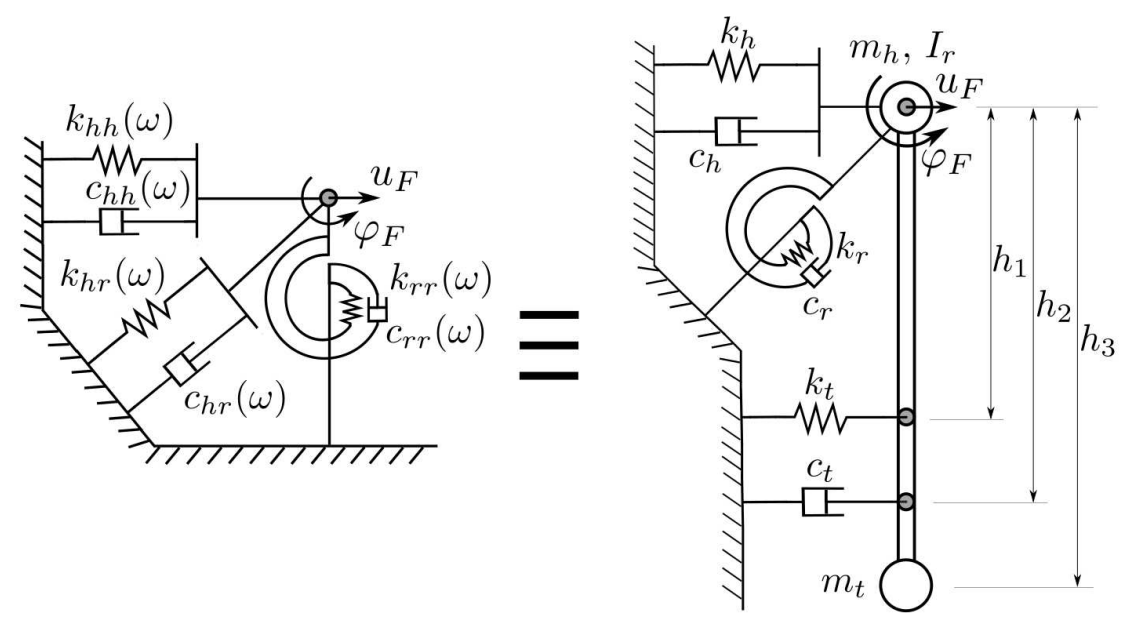

Figure 3: LPMs schemes: a) consistent [33, 34, 35], b) simplified [36].

$$
\begin{aligned}
& {\left[\begin{array}{cc}
\hat{Z}_{h h}(\omega) & \hat{Z}_{h r}(\omega) \\
\hat{Z}_{r h}(\omega) & \hat{Z}_{r r}(\omega)
\end{array}\right]=\left[\begin{array}{cc}
k_{h}+k_{t} & k_{t} h_{1} \\
k_{t} h_{1} & k_{r}+k_{t} h_{1}^{2}
\end{array}\right]-\omega^{2}\left[\begin{array}{cc}
m_{h}+m_{t} & m_{t} h_{3} \\
m_{t} h_{3} & I_{r}+m_{t} h_{3}^{2}
\end{array}\right]+} \\
& +\mathrm{i} \omega\left[\begin{array}{cc}
c_{h}+c_{t} & c_{t} h_{2} \\
c_{t} h_{2} & c_{r}+c_{t} h_{2}^{2}
\end{array}\right]
\end{aligned}
$$

where all real and imaginary terms are parabolic and linear functions of omega, respectively.

The presence of the additional mass $m_{t}$ at a certain depth makes the model able to capture the coupled roto-translational dynamic response of the foundation, and contrary to the approach adopted for the consistent LPM, the fitting process of the translational, rotational and coupled impedance functions are made simultaneously. Also, thanks to the rigid link, the dynamic stiffness matrix of the LPM can be condensed on the degrees of freedom of the master node on the foundation-structure interface (i.e. there are no hidden nodes). Another advantage of this configuration is the possibility of an easy implementation in many commercial software. However, conventional software usually do not allow the introduction of negative values of masses, dampers and springs, so, in order to maintain this advantage in the simplified model, all real constants of the scheme are forced to be positive, with the only exception of the different lengths of the rigid link $\left(h_{1}, h_{2}\right.$ and $\left.h_{3}\right)$. The resulting parabolic horizontal and rocking stiffness functions are concave due to the positive values of the masses, with a positive static value. On the contrary, the crossed stiffness can be convex and the static value can be positive or not, depending on the sign of $h_{1}$ and $h_{3}$. Furthermore, since the problem condensation produces non-diagonal terms in the mass matrix of the LPM, it may be convenient to physically model the rigid link and the eccentric mass since non-diagonal mass matrices are often not implemented in commercial structural analysis software.

In the case of this simplified approach, the LPM parameters that appear in Equation (3) are defined as: 


$$
\begin{aligned}
{\left[\begin{array}{ll}
\bar{k}_{h h} & \bar{k}_{h r} \\
\bar{k}_{r h} & \bar{k}_{r r}
\end{array}\right] } & =\left[\begin{array}{cc}
k_{h}+k_{t} & k_{t} h_{1} \\
k_{t} h_{1} & k_{r}+k_{t} h_{1}^{2}
\end{array}\right] \\
{\left[\begin{array}{ll}
\bar{c}_{h h} & \bar{c}_{h r} \\
\bar{c}_{r h} & \bar{c}_{r r}
\end{array}\right] } & =\left[\begin{array}{cc}
c_{h}+c_{t} & c_{t} h_{2} \\
c_{t} h_{2} & c_{r}+c_{t} h_{2}^{2}
\end{array}\right] \\
{\left[\begin{array}{ll}
\bar{m}_{h h} & \bar{m}_{h r} \\
\bar{m}_{r h} & \bar{m}_{r r}
\end{array}\right] } & =\left[\begin{array}{cc}
m_{h}+m_{t} & m_{t} h_{3} \\
m_{t} h_{3} & I_{r}+m_{t} h_{3}^{2}
\end{array}\right]
\end{aligned}
$$

In both approaches, the different LPM constants $\mathbf{x}$ that define the model are found through a non-linear least square fitting procedure of the response of the LPM to the reference impedance functions (computed as mentioned in Section 3.1), as follows:

$$
\min _{\mathbf{x}}\|(\hat{Z}(\mathbf{x}, \omega)-Z(\omega)) \Psi(\omega)\|_{2}^{2}=\min _{\mathbf{x}} \sum_{i}\left(\left(\hat{Z}\left(\mathbf{x}, \omega_{i}\right)-Z\left(\omega_{i}\right)\right) \Psi\left(\omega_{i}\right)\right)^{2}
$$

In both cases, the initial fitting frequency range is $0-20 \mathrm{~Hz}$. However, due to its nature, a bad fitting of damping coefficients in the lower frequency range is obtained, even if a higher order LPM is used, when trying to fit functions obtained with the hysteretic soil damping model. In this case, the reference damping functions tend to infinity when approaching a zero frequency; a behaviour that cannot be reproduced by the LPM models (see Paronesso and Wolf [37]). Consistent LPM functions are symmetric with respect to frequency, which implies a null slope at the static frequency, fact that strongly influences the result of the fitting procedure. Simplified LPM functions, on the other hand, can not catch fluctuations of the reference impedance functions, so that the models' ability to reproduce the soil-foundation impedances in a selected frequency range strongly depends on their variability with frequency. For the present study, the variability associated to pile-to-pile resonant peaks tends to appear at frequencies above the superstructure fundamental frequencies considered herein. It is also worth mentioning that the influence of the static stiffness in the problem at hand makes it mandatory to give somehow more importance to the lower frequency range. For this reasons, in order to balance the bad fitting at lower frequency range, a frequency-dependent weight function $\Psi$ is employed. The weight functions are defined as follows

$$
\begin{aligned}
& \Psi_{\text {consistent }}(\omega)=\left(1+\left(c_{1} \frac{c_{s}}{d} \omega\right)^{c_{2}}\right)^{-c_{3}} \\
& \Psi_{\text {simplified }}(\omega)=H\left(f_{c}\right)
\end{aligned}
$$

with $c_{1}=4.0, c_{2}=2.0$ and $c_{3}=2.0$ in the first function $[33,34], f_{c}$ a cut-off frequency set at $6 \mathrm{~Hz}$, and $H()$ being the unit step function. With these expressions, represented in Figure 4 for reference, substantially more importance is given to the frequency range were the superstructure fundamental frequencies reside, and significantly more accurate structural responses are obtained.

\subsection{Parametric analysis}

The combination of the superstructures, foundation layouts, soil deposits, soil damping models and seismic actions described above defines the set of configurations whose seismic response is analysed in the present study. In short, four different bridge superstructures characterized by different fundamental periods $\left(T_{n}=0.2 \mathrm{~s}, T_{n}=0.5 \mathrm{~s}, T_{n}=1.0 \mathrm{~s}\right.$ and $\left.T_{n}=1.5 \mathrm{~s}\right)$ can be founded on six groups of piles layouts on the foundation $(2 \times 2$ and $3 \times 3$ pile groups with spacings of 3,5 and $7 \mathrm{~m})$ embedded on two different deposits (D and C), subjected to seven different seismic signals for each ground type. 
At the same time, two different soil damping models are considered (hysteretic and Biot's), and the problems are solved by means of three different methodologies; the frequency-domain method of response, and two time-stepping approaches in which the foundation behaviour is modelled through the different LPMs described above (simplified and consistent). Overall, also including FB cases, a total of 2072 different cases have been analysed. The results of this large set are synthesized below.

\subsection{Error measurement}

The large number of cases involved in the study requires a way to synthesize the results so that the influence of the different parameters can be evaluated. Firstly, the effectiveness of the different LPMs when used to obtain the system response in time domain is measured using Equation (10), where $x$ stands for the degree of freedom under consideration $\left(\varphi_{S}, u_{F}\right.$ or $\left.\varphi_{F}\right)$, "sdm" stands for the soil damping model considered (hysteretic or Biot's), and "lpm" stands for the LPM used (simplified or consistent). This measure of the differences takes into account the entire earthquake duration of the seismic signal, being $P$ the total amount of time steps in the time history. Results obtained through the frequency domain method of response are chosen as reference.

$$
\varepsilon_{x_{\mathrm{lpm}}}^{\mathrm{sdm}}=\sqrt{\frac{\sum_{i=0}^{P}\left(x_{\mathrm{lpm}, i}^{\mathrm{sdm}}-x_{\text {freq. domain }, i}^{\text {sdm }}\right)^{2}}{\sum_{i=0}^{P}\left(x_{\text {freq. domain }, i}^{\text {sdm }}\right)^{2}}}
$$

On the other hand, the influence of the soil damping model is quantified using Equation (11). Again, this measure of the differences takes into account the entire earthquake duration of the seismic signal. In this case, all responses are computed through the frequency domain method of response analysis. Results corresponding to the hysteretic soil damping model are chosen as reference.

$$
\chi_{x}=\sqrt{\frac{\sum_{i=0}^{P}\left(x_{\text {Biot }, i}-x_{\text {hysteretic }, i}\right)^{2}}{\sum_{i=0}^{P} x_{\text {hysteretic }, i}^{2}}}
$$

\section{Results}

\subsection{Influence of soil damping model on impedance and kinematic inter- action functions}

As a first step, impedance and kinematic interaction (KI) functions in the frequency domain must be computed in order to build the different subestructuring models used in the analyses. When doing so, soil damping is considered using either Biot's or hysteretic damping, so that its influence on the overall response can later be analysed. In order to illustrate the effects on impedance functions of assuming one damping model or the other, horizontal, rocking and crossed-coupled impedance functions $\left(Z_{i j}(\omega)=k_{i j}(\omega)+\mathrm{i} \omega c_{i j}(\omega)\right)$ are presented in Figure 4 in terms of stiffness $\left(k_{i j}(\omega)\right)$ and damping $\left(c_{i j}(\omega)\right)$ functions, for both hysteretic and Biot's models (see solid and dashed black lines, respectively), for the specific case of a $2 \times 2$ pile group, embedded in a type D ground and with a pile-to-pile spacing $s=7 \mathrm{~m}$.

The main difference between both models lies in the damping coefficient at very low frequencies. As anticipated, the hysteretic model produces a damping function such that the imaginary part of the impedance function is non-zero at $\omega=0$, while Biot's model leads to a finite damping 

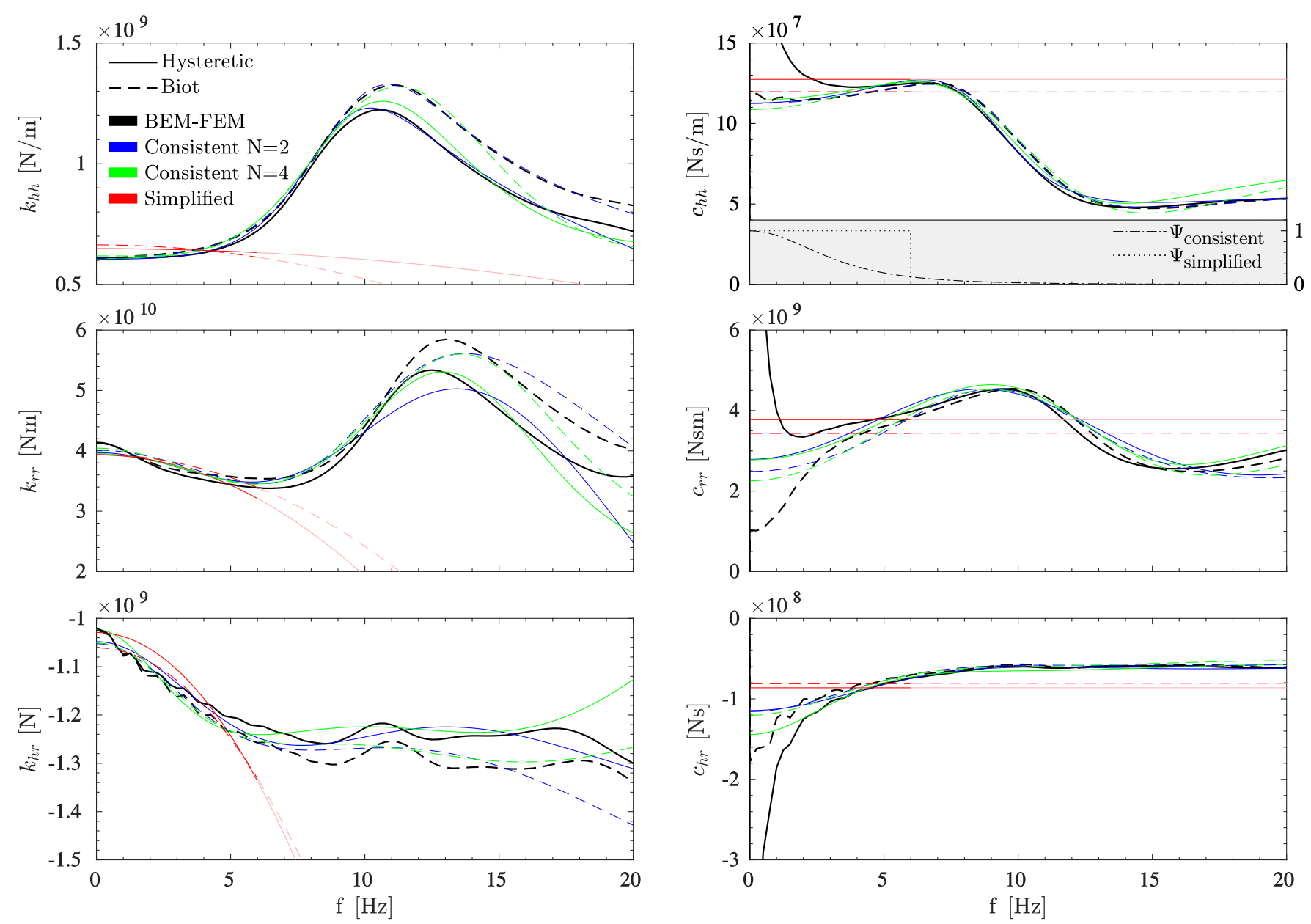

Figure 4: Illustration of the computed and fitted impedance functions for one of the configurations analyzed $(2 \times 2, s=7 \mathrm{~m}$ foundation layout in ground type D). Continuous and dashed black lines represent impedance functions assuming hysteretic and Biot's damping models, respectively; blue and green lines represent fitted functions using consistent LPM with orders $N=2$ and $N=4$, respectively; red lines represent fitted functions using simplified LPM; and $\Psi$ represents the weight functions used by the LPM approaches (defined in Equations (9a) and (9b)).

function at zero, which implies an imaginary part of the impedance function that vanishes for a static situation. On the contrary, the damping functions obtained with both models are almost coincident for frequencies above $5 \mathrm{~Hz}$. Stiffness functions, on the other hand, coincide at low frequencies, but differ at frequencies above $10 \mathrm{~Hz}$, with Biot's model leading to larger stiffness values.

Kinematic interaction factors are also affected by the soil damping model chosen for the analysis. In order to illustrate the magnitude of this effect, Figure 5 presents modulus and phase of the translational and rotational kinematic interaction factors obtained for the same configuration presented above, together with the normalized mean Fourier amplitude spectrum of the ground motion, scaled for superstructure $3\left(T_{n}=1.0 \mathrm{~s}\right)$. The classical hysteretic soil damping model predicts a slightly larger filtering effect for the translational kinematic interaction factors, with a larger induced rotation of the foundation for frequencies below $11 \mathrm{~Hz}$. On the contrary, Biot's soil damping model predicts larger rotations for frequencies above $11 \mathrm{~Hz}$. On the other hand, the phase of the rotational kinematic interaction function illustrates very clearly the incoherences arising from the use of the standard hysteretic damping model, with a non-zero phase delay for the static situation. Apart from this, the phase of the translational motion is larger when the hysteretic model is assumed.

In any case, the relevance of the differences between impedance or kinematic interaction functions 

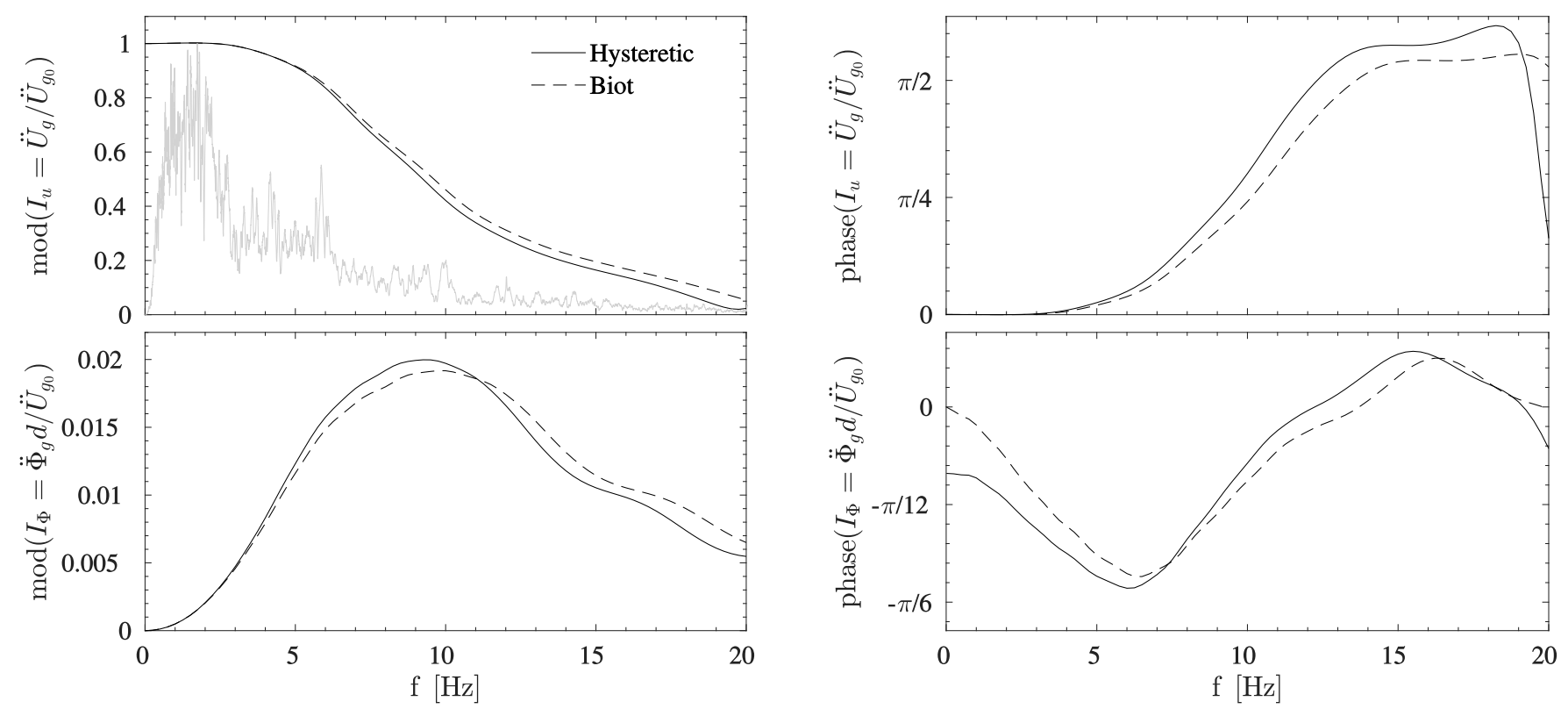

Figure 5: Illustration of the computed kinematic interaction factors for $2 \times 2, s=7 \mathrm{~m}$ foundation layout in ground type D. Continuous and dashed black lines represent kinematic interaction factors assuming hysteretic and Biot's damping models, respectively; the gray line represents normalized mean Fourier amplitude spectrum of the ground motion, scaled for superstructure $3\left(T_{n}=1.0 \mathrm{~s}\right)$.

obtained from one damping model or the other will depend on other aspects such as the magnitude of the SSI effects, or the natural frequencies of the superstructure. Depending on the properties of the superstructure, foundation and/or seismic input, certain differences in one function or frequency range can be important or totally anecdotal. Therefore, the influence of the damping model must also be assessed analysing the magnitude of the differences arising in the superstructural response due to this aspect. For this reason, subsequent sections will focus not on impedance or kinematic interaction functions, but on differences on the response at the superstructure. As an illustration example in this case, the effect of the differences highlighted above between impedances and kinematic interaction functions for the particular case of the $2 \times 2$ pile group, will be examined below.

Firstly, the small differences appreciated in the kinematic interaction factors between damping models translate to even smaller differences when observing the translational kinematic input motion $\ddot{u}_{g}$ shown in Figure 6 together with the free field ground motion $\ddot{u}_{g_{0}}$ and the rotational kinematic input motion $\ddot{\varphi}_{g}$ for the same specific case subjected to 429-111 earthquake (see Table 3) and scaled for superstructure $3\left(T_{n}=1.0 \mathrm{~s}\right)$. For instance, the magnitude of the differences arising at one of the most significant peaks of the signal (at $t=9.915 \mathrm{~s}$ ) can be quantified in $1.6 \%$ and $8.3 \%$ for the translational and the rotational kinematic interaction functions, respectively. It is worth noting that, as illustrated in Figure 5, the predominant frequencies of the ground motion are located in the range where the filtering produced by the foundation is still not significant.

On the other hand, Figure 7 presents results corresponding to the response of the particular case of the $T_{n}=1.0 \mathrm{~s}$ superstructure (see Table 3 ), founded on the $2 \times 2$ pile group on soil deposit $\mathrm{D}$, whose impedance and kinematic interaction functions were presented above, and subject to earthquake 429-111, so that the corresponding FIM is the one shown in Figure 6. The response of the system in terms of the three degrees of freedom defined above (translation $u_{F}$ and rotation $\varphi_{F}$ of the foundation, and relative rotation of the pier with respect to the foundation $\varphi_{S}$ ) is computed by solving Equation (1) directly in the frequency domain and considering both hysteretic and Biot's models (see black lines). FB response is also presented for reference. The quantification, in this particular case, of the differences between results obtained from both damping models, and the error 

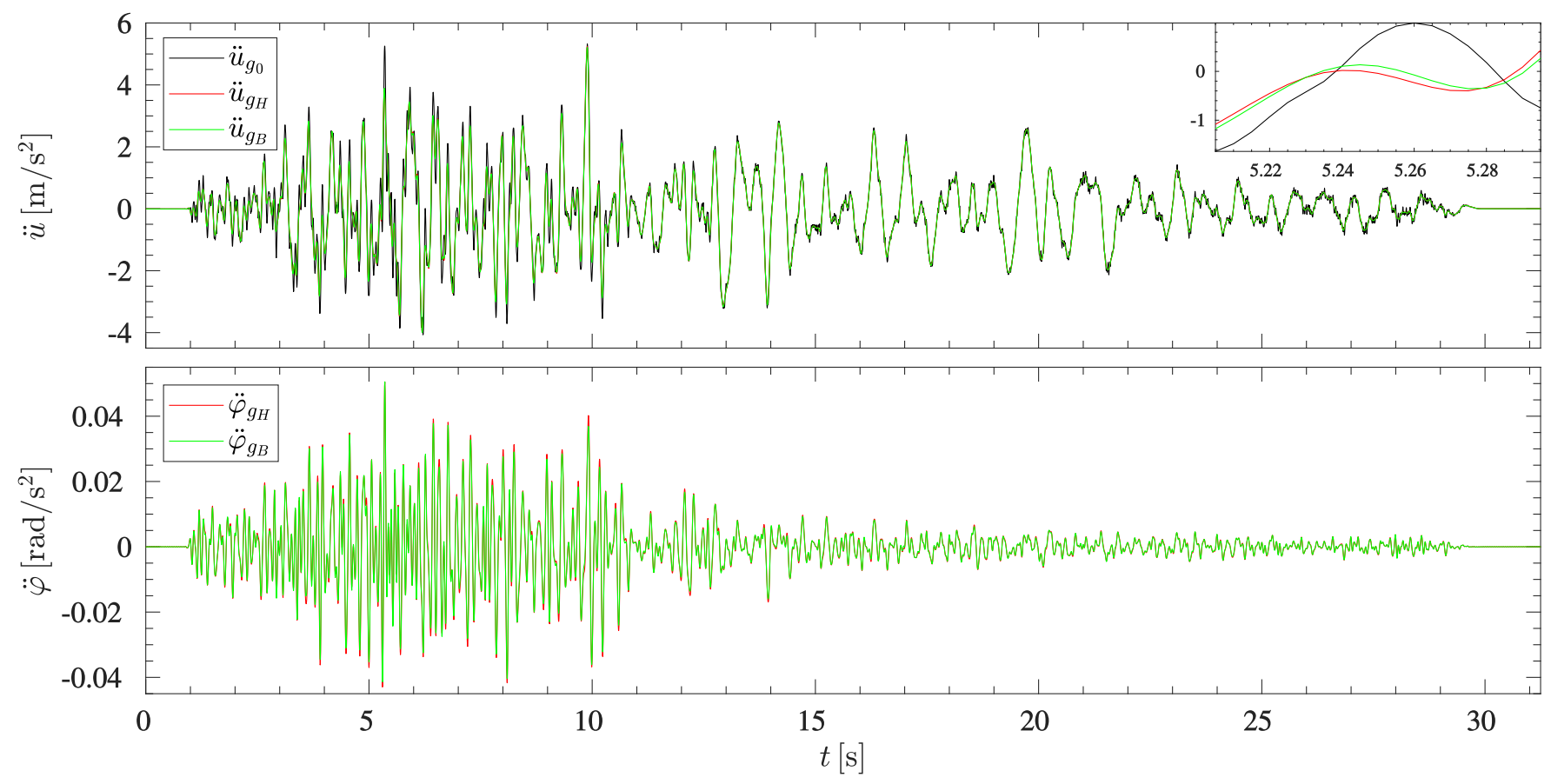

Figure 6: Time history of 429-111 earthquake acceleration (Table 3), scaled for superstructure 3 $\left(T_{n}=1.0 \mathrm{~s}\right)$; and FIM derived from soil-foundation kinematic interaction analysis. $2 \times 2, s=7 \mathrm{~m}$ foundation layout in ground type D.

\begin{tabular}{c|c|ccc|crr} 
& $\chi$ & \multicolumn{3}{|c|}{$\varepsilon$ (hysteretic) } & \multicolumn{3}{c}{$\varepsilon$ (Biot) } \\
& & Cons. N=2 & Cons. N=4 & Simpl. & Cons. N=2 & Cons. N=4 Simpl. \\
\hline$\varphi_{S}$ & 0.136 & 0.079 & 0.087 & 0.018 & 0.056 & 0.040 & 0.095 \\
$u_{F}$ & 0.168 & 0.099 & 0.102 & 0.055 & 0.063 & 0.049 & 0.156 \\
$\varphi_{F}$ & 0.169 & 0.102 & 0.112 & 0.037 & 0.071 & 0.050 & 0.111
\end{tabular}

Table 5: Differences between damping models and errors committed by using the different LPM approaches in the results shown in Figure 7.

committed when using the LPM approaches is presented in Table 5 for illustration purposes $(\chi$ values). The difference between fixed and flexible base responses illustrates the relevance of the SSI effects. On the other hand, differences between the two soil damping models are much less important, being the influence of the soil damping model on the response of the superstructure smaller than that on the response at the foundation level.

\subsection{Parametric analysis of the system seismic response}

This section presents the results of the parametric analyses studying the influence of the soil damping model and of the LPM, on the seismic response of the system. Given the large number of cases under analysis, the results are synthesized and presented in different ways in order to highlight different effects. For a better understanding of such statistical analysis and of the methodology used to produce the base results, the response of the specific configuration tackled in the previous section is first presented in detail in terms of its time response.

The second step in every analysis (after having computed impedance and kinematic interaction functions) is obtaining the LPM that represents the compliance of the foundation under consideration. For the specific $2 \times 2$ pile group discussed above, embedded in the soil deposit $\mathrm{D}$ and with a pile 

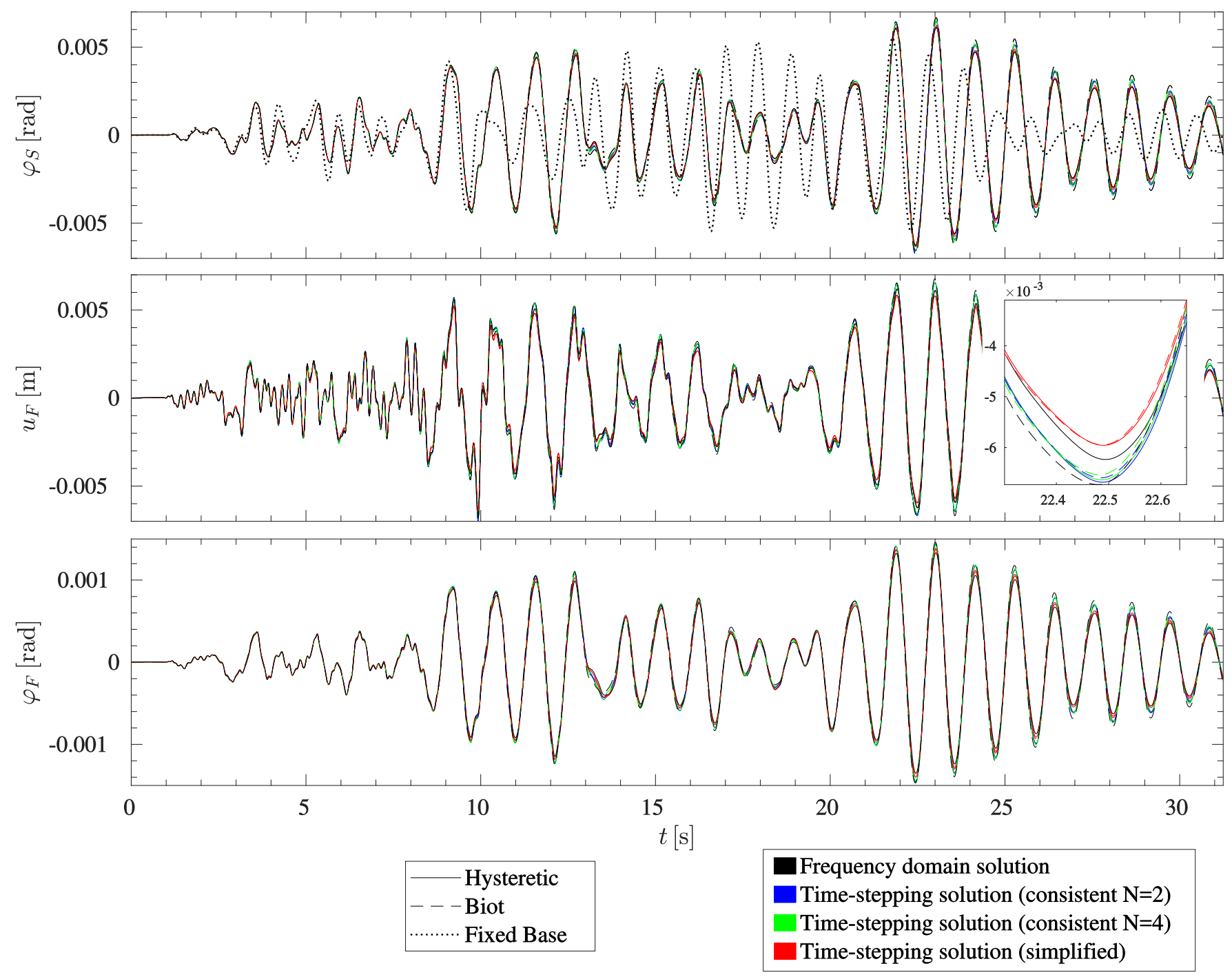

Figure 7: Time history of superstructure and pile cap degrees of freedom. Particular case of $2 \times 2$, $s=7 \mathrm{~m}$ foundation layout in ground type D (impedance functions and kinematic interaction factors shown in Figures 4 and 5 respectively) using superstructure $3\left(T_{n}=1.0 \mathrm{~s}\right)$ and earthquake $429-111$ (Table 3).

spacing of $s=7 \mathrm{~m}$, Figure 4 presents three different sets of fitted impedance functions together with the original stiffness and damping functions, already discussed above, computed with the BEM-FEM approach for both soil damping models. One of the fitted sets is obtained through the simplified LPM proposal, while the two other sets are obtained through the consistent LPM approach with two and four internal degrees of freedom $(N=2$ and $N=4)$, respectively.

The LPMs obtained through the consistent approach are able to fit the stiffness functions very accurately along the whole frequency range, with errors increasing with frequency, as can be expected taking into account the weighting function that has been used. The fitting of the damping functions is also quite remarkable except for the very low frequencies when fitting the hysteretic impedances. In this case, the damping functions tend to infinity as frequency approaches zero, a behaviour that the LPMs are unable to reproduce because consistent LPM functions are symmetric, and therefore their slope at frequency zero must be horizontal. It is also worth to highlight that the use of more than two internal degrees of freedom does not always improve this fitting and, in general, does not improve the overall result, reason why configurations with $N=2$ will be used in the following.

The fitted functions obtained using the simplified LPM approach are quite different and, a priori, 
do not fit the original functions very well, although it will be shown later that, in general, they are able to produce very good results. Simplified LPM stiffness functions are parabolic, so can not accommodate complex phenomena as the group effect presents in this illustrated configuration. However, the fitting at the low frequency range, prevalent when computing the response of the system, can be considered adequate, especially for the rocking component. The resulting damping function is frequency-independent, and represents the average damping in the frequency range of interest.

The fitted LPM functions for each configuration are used to compute the response of the system using a time-domain step-by-step scheme. Figure 7 presents a comparison between the time-history response of the system computed directly in the frequency domain or in time domain with both LPM approaches, for the case discussed above and for both soil damping models. From an engineering point of view, both LPMs are indeed able to simulate well the soil-foundation compliance since quite small differences arise between any one of them and the reference frequency domain results. In fact, the errors induced by approximating the impedance functions through the LPM approaches are smaller than the differences found between the responses of the system assuming one damping model or the other $(\varepsilon<\chi$, shown in Table 5).

However, for this particular case, a significant aspect is worth of notice: when a hysteretic damping model is assumed for the soil, the overall error obtained by using the simplified LPM is smaller than the one resulting from the use of the consistent $\operatorname{LPM}\left(\varepsilon_{\text {simplified }}^{\mathrm{H}}<\varepsilon_{\text {consistent }}^{\mathrm{H}}\right)$ for all the degrees of freedom of the superstructure. Taking into account that the structural response is mainly dominated by the fundamental frequency of the compliant system, this result should be related to a lower accuracy of the consistent LPM, with respect to the simplified one, in reproducing the soil-foundation impedances around that fundamental frequency. Taking a look at the hysteretic rocking damping function at the $\mathrm{CB}$ fundamental frequency of this system $\left(0.89 \mathrm{~Hz}\right.$ for the $f_{n}=1 \mathrm{~Hz}$ superstructure) on Figure 4, the values obtained with the simplified approach around this point are closer to the reference function (that tends to infinity at low frequencies) than the ones obtained from the consistent approach. The opposite occurs assuming Biot's damping model, $\varepsilon_{\text {simplified }}^{\mathrm{B}}>\varepsilon_{\text {consistent }}^{\mathrm{B}}$. In this case, for which the reference function is perfectly bounded, the fitting obtained using the consistent approach is much more satisfactory. It is worth noting that, as observed before, the use of a higher number of internal degrees of freedom in the consistent LPM does not always lead to better results. For instance, for a hysteretic soil damping model, the error is larger for $N=4$ than for $N=2$ (see Table 5).

The following sections will look at how all these observations are affected by the properties of the system under study. First of all, the magnitude of the SSI effects will be evaluated (through Figures 8 and 9), given that all the aspects discussed here are relevant only when this phenomenon is important (stiff soil-foundation systems will be less affected by errors in the fitted functions because of the small relevance of considering a CB system). Then, results will be synthesized to study the influence of ground type, superstructure fundamental frequency and pile foundation configuration.

Thus, Figure 8 presents the contribution of the different degrees of freedom to the horizontal displacement of the bridge deck for all foundation cases considered in this study. For each possible configuration, the mean maximum bridge deck displacement over the seven earthquakes is presented in one vertical bar. Each of these bars is divided in three sections, each one representing the contribution of a different degree of freedom (foundation rotation, foundation displacement and superstructure rotation). To represent the contribution of both rotations, $\varphi_{S}$ and $\varphi_{F}$, to the lateral displacement of the bridge deck, they are respectively multiplied by the length from the $S$ node (base of the pier) to the bridge deck centroid $D\left(h_{S}=h_{p}+h_{c}+h_{d}\right)$, and the length from the $F$ node (ground level) to the bridge deck centroid $D\left(h_{F}=h_{f}+h_{p}+h_{c}+h_{d}\right)$, Figure 1. At the same time, these bars are grouped in sets of threes, each one in the set representing the results obtained following the frequency-domain method of response analysis, or a time-stepping procedure in which the soil-foundation system is obtained using a consistent $(\mathrm{N}=2)$ or a simplified LPM approach. Finally, a different set of bars is 

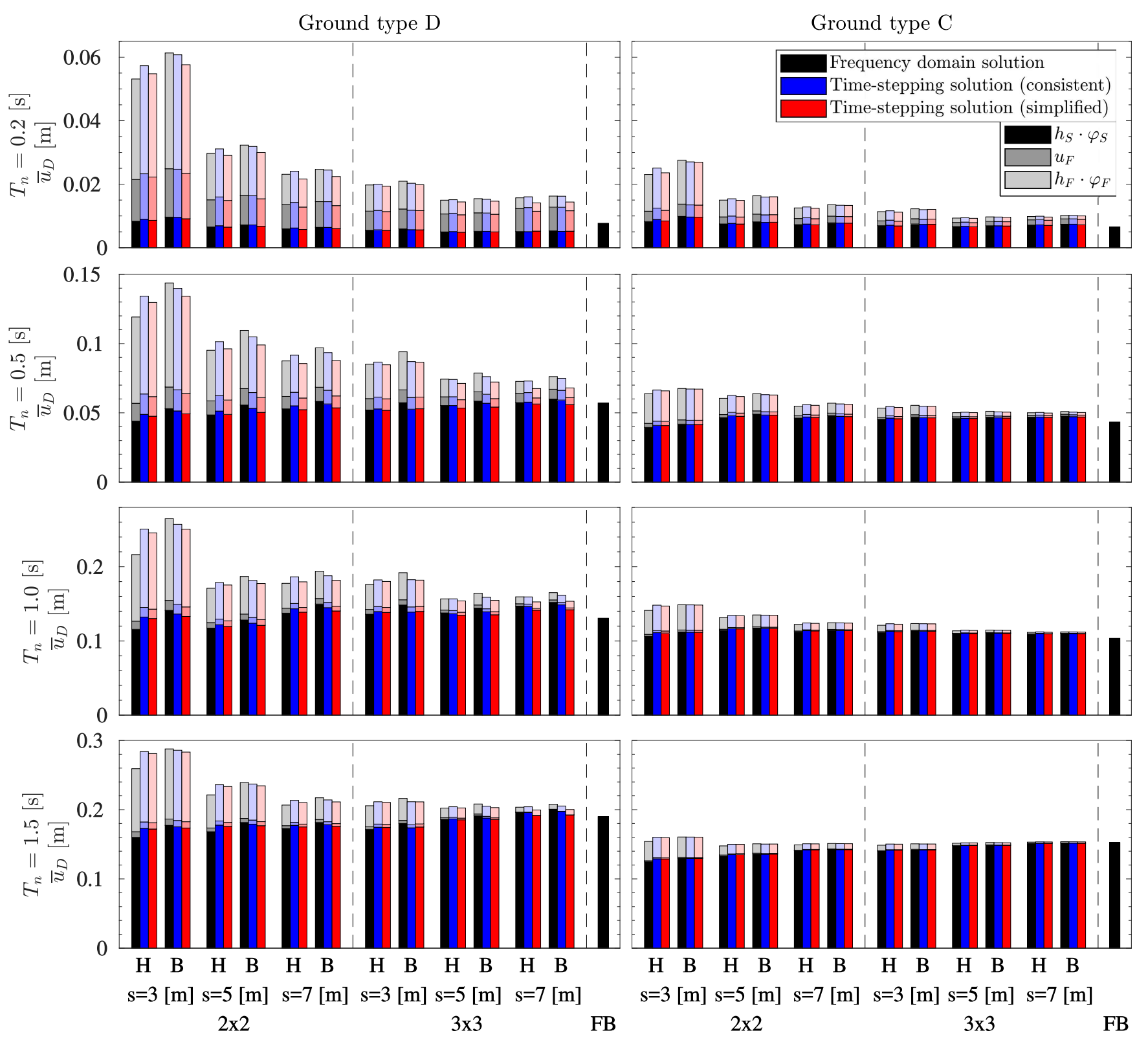

Figure 8: Contribution of the different degrees of freedom to the mean maximum lateral displacement of the bridge deck $u_{D}$ (FB: fixed-base, H: hysteretic damping model and B: Biot's damping model).

presented for each one of the configurations considered for a different superstructure, foundation and soil damping model. FB response is also included for reference. Note that the vertical scales used for different superstructures (plots in different rows within the figure) change, with increasing ranges for increasing fundamental periods.

From the comparison between FB and CB models, it becomes clear that SSI phenomena is relevant in most configurations. It is also worth noting that deck displacements are always smaller if a hysteretic damping model is considered due to its higher foundation damping at low frequencies.

Bridge deck maximum motions arising in the stiffest (and shortest) configurations are much smaller than those arising in the softer (and taller) cases, but the relative contribution of pile cap degrees of freedom is much more important in the first situation. On the other hand, for any specific superstructure, deck displacements increase by reducing the pile spacing or the number of piles constituting the group, as the stiffness of the soil-foundation system decreases and, as a consequence, the contribution of the foundation displacement and rocking increases. As expected, the contribution of the foundation rocking is very significant for the soft soil D and tight foundations. 

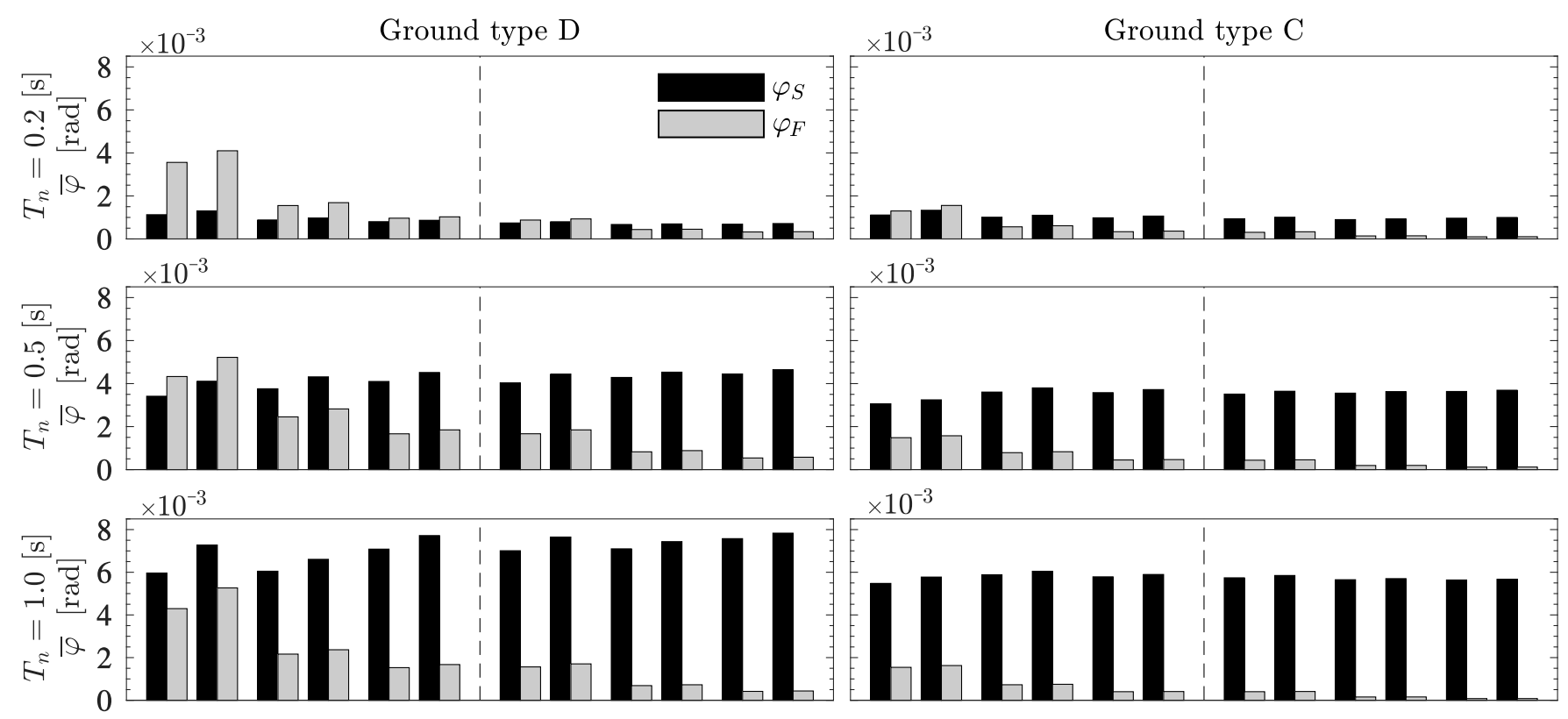

$\times 10^{-3}$

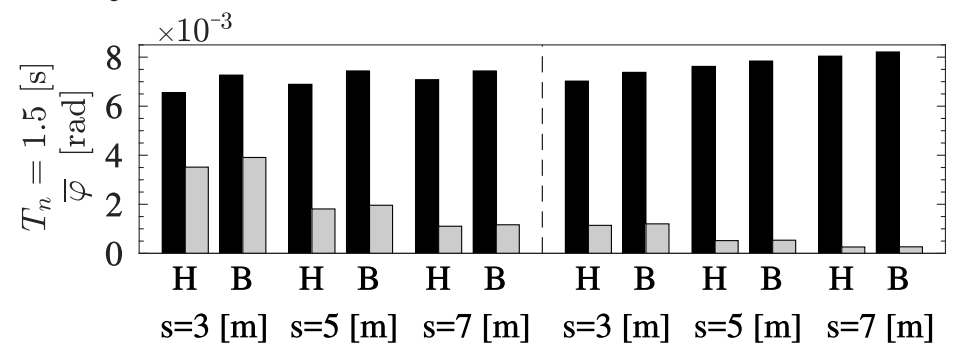

$2 \times 2$

$3 \times 3$
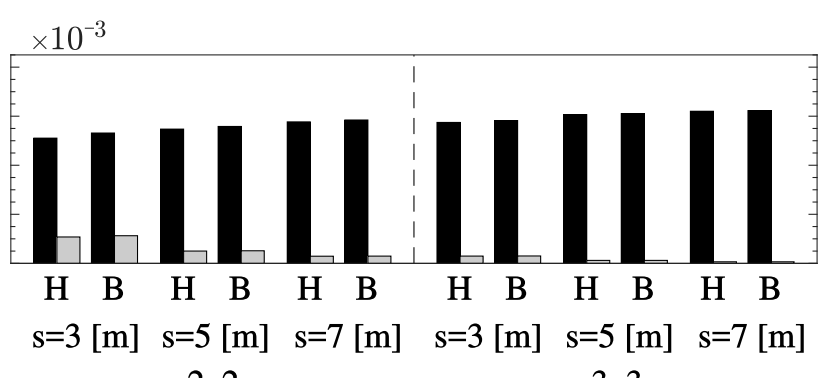

Figure 9: Comparison between the mean maximum magnitudes of foundation rotation and superstructure relative rotation using frequency domain approach (FB: fixed-base, H: hysteretic damping model and B: Biot's damping model).

In fact, for the stiffest superstructures $\left(T_{n}=0.2 \mathrm{~s}\right.$ and $\left.T_{n}=0.5 \mathrm{~s}\right)$ on $2 \times 2$ pile groups, it represents more than $50 \%$ of the total motion. This can be observed more clearly in Figure 9, where the mean maximum magnitudes of foundation rotation and superstructure relative rotations computed in frequency domain are compared in a format similar to that of Figure 8. The relative importance of the foundation rotation over the superstructure relative rotation depends not only on the soil-foundation system compliance, but also on the stiffness (and height) of the superstructure. The relative rotation of the superstructure is prevalent for the three softest structures $\left(T_{n}=0.5 \mathrm{~s}, T_{n}=1.0 \mathrm{~s}\right.$ and $\left.1.5 \mathrm{~s}\right)$ with the only exception of the $T_{n}=0.5 \mathrm{~s}$ superstructure on the softest foundation. On the other hand, foundation rotation is prevalent in the case of the stiffest superstructure on a soft foundation. In this regard, it is interesting to note that, for a given foundation and ground type, the magnitude of the foundation rotations tends not to change significantly for different superstructures (while, as expected, the contribution of the superstructure relative rotation increases significantly with softer superstructures). It is also worth noting that Biot's soil damping model (which predicts a slightly softer foundation rocking response) tends to anticipate larger foundation rotations and, therefore, larger superstructure deformations. 


\subsection{Parametric analysis of the influence of different aspects on the ac- curacy of the responses computed using LPMs}

Figure 8 shows how the three solution approaches adopted (frequency-domain solution with the original impedance functions, and the two time-stepping approaches using two different LPM approaches to model the foundation response) provide slightly different solutions. If hysteretic damping is assumed, the time-stepping approaches tend to predict bridge maximum motions that are slightly larger than those obtained from the frequency-domain solution, being this the situation in $77.5 \%$ of the cases analysed. On the contrary, when Biot's damping is assumed, the bridge deck maximum lateral displacements predicted by the time-stepping approaches are smaller than those of the frequency-domain solution in almost all cases (93.2\% of the cases). This is so because the responses computed directly in the frequency-domain are much more sensitive to changing from Hysteretic to Biot's damping than time-domain approaches (see Figure 8), as part of the information is lost during the fitting process of definition of the LPM sub-system. However, in order to evaluate which of the four considered approaches could be considered as more conservative in terms of structural integrity, the variable of interest should be the peak superstructure relative rotation $\varphi_{S}$. When the result obtained for this magnitude is compared among the four possible approaches, the assumption of Biot's damping together with the adoption of the consistent lumped parameter model provides the highest relative rotations in $72.9 \%$ of the cases which indicates that such approach could be adopted as the most conservative option.

In order to assess the reliability of the two LPM approaches when used in this kind of problems, it is mandatory though to quantify these differences, and to study how they are affected by different aspects of the problem. For this reason, the next sections will quantify the errors committed, with respect to the reference frequency-domain approach, as a function of ground type, superstructure configuration and foundation layout. Errors between each time-stepping procedure and the reference frequency-domain solution $(\varepsilon)$ and differences between soil damping models $(\chi)$ will be presented in standard box-and-whisker diagrams (Figures 10 to 13), with results grouped in the relevant way for each aspect. In all cases, differences between damping models are presented in grey tones on the left side of the plots, while errors committed when using one of the lumped parameter equivalent models is presented in blue (consistent, $N=2$ ) and red (simplified) colors. Results obtained from hysteretic and Biot's damping assumptions are presented separately in two adjacent subplots. All cases are plotted as a set of three box-and-whisker representation, each of them representing the error or difference measured on one of the three degrees-of-freedom of the system, in different levels of darkness (as shown in the legends).

\subsubsection{Influence of ground type}

Figure 10 presents errors and differences for all configurations grouped according to the ground type D (left) or C (right), respectively. Errors arising in systems founded on type C grounds (medium stiffness soils) tend to be significantly smaller than those observed on type D grounds (soft soils). This is due to two main causes: a) as expected, the magnitude of the SSI effects is much larger for the latter, i.e., the contribution of the foundation response, where the approximations related to the LPMs lie, is more relevant; and b) changing from type C to D grounds, resonances peaks that characterise the impedances trends moves towards lower frequencies and impedances present more regular trends that can be better approximated by LPMs. From an engineering point of view, errors committed are very low, specially in type $\mathrm{C}$ grounds, and are largely compensated by practical advantages deriving from the use of the LPM approaches (i.e. possibility of using time-domain solution approaches, and of using dedicated computer software for structural analysis that usually perform time domain analysis).

Regarding the influence of soil damping model, the overall errors obtained by considering the consistent and simplified LPMs are very similar when the hysteretic model is assumed, though for 

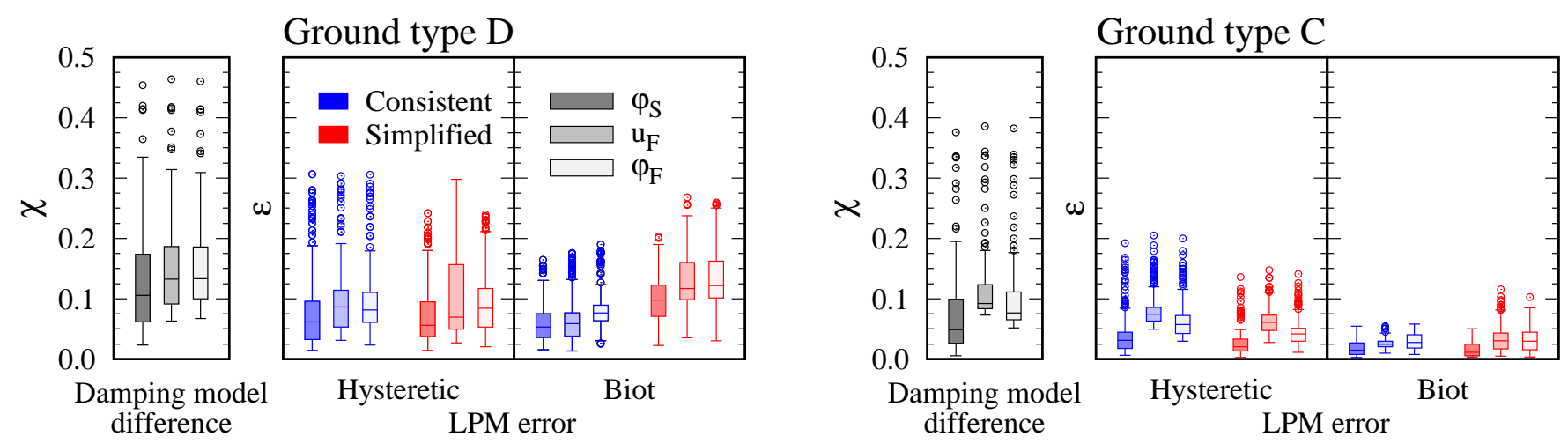

Figure 10: Differences due to the use of hysteretic or Biot's soil damping model, and errors due to the use of LPM approaches. Influence of ground type.

medium stiffness soil the error deriving from the use of the simplified LPM is slightly lower than that of the consistent LPM. For Biot's damping model, on the other hand, the overall error obtained with the consistent LPM is always lower than that resulting from the simplified option. From an engineering point of view, the median errors resulting from the LPM approach are limited (always below $\varepsilon=0.1$, except for the simplified option in the case of Biot's damping model in soft soils). However, errors are significantly scattered around the median values, except for the case of systems on type $\mathrm{C}$ grounds considering Biot's damping model.

It is worth noting that differences in the seismic response of the system deriving from the use of one damping model or the other are of the same order of magnitude (though always slightly higher) than the errors produced by the approximation of the impedance functions, which suggests that the errors committed when using any of these LPMs to represent the foundation are less relevant than the effect of the simplifying assumptions of the original model.

\subsubsection{Influence of superstructure fundamental periods}

Figures 11 and 12 synthesizes errors and differences for all configurations grouped according to the superstructure involved in each case, with the first figure referring to structures on soft soils, and the second to medium soils. In general, overall errors obtained by considering the consistent and simplified LPMs are very similar to each other if soil hysteretic damping is assumed, although maximum errors committed with the first option tend to be largest in stiff structures on medium soils, while those committed with the second option are the ones that tend to be larger for stiff structures on soft soils. With the exception of these specific cases, no advantage is clearly evident from the use of one LPM or the other. On the other hand, when assuming Biot's damping model, the overall errors committed due to the use of the consistent LPM tend to be lower than that resulting from the simplified model.

The magnitude of the errors is almost independent of the fundamental period of the superstructure (the median value is always around $0.08<\varepsilon<0.12$ ). This observation can be explained by the fact that soil-foundation impedances are well reproduced by both LPMs in the frequency range covering the fundamental superstructure frequencies. The soil-foundation impedances do not present articulated trends with frequency, namely no resonance peaks occur in the frequency range where fundamental frequencies lie (except for the $3 \times 3, s=7 \mathrm{~m}$ specific case) that cannot be captured by the simplified LPM. When soil hysteretic damping is assumed, the LPM errors observed for superstructures with the smallest fundamental period $\left(T_{n}=0.2 \mathrm{~s}\right)$ are much smaller than those obtained for the rest of superstructures because their fundamental frequencies go beyond the frequency range for which damping coefficients are unbounded.

Regarding soil type C, as already observed, errors are much lower than those arising for soil 

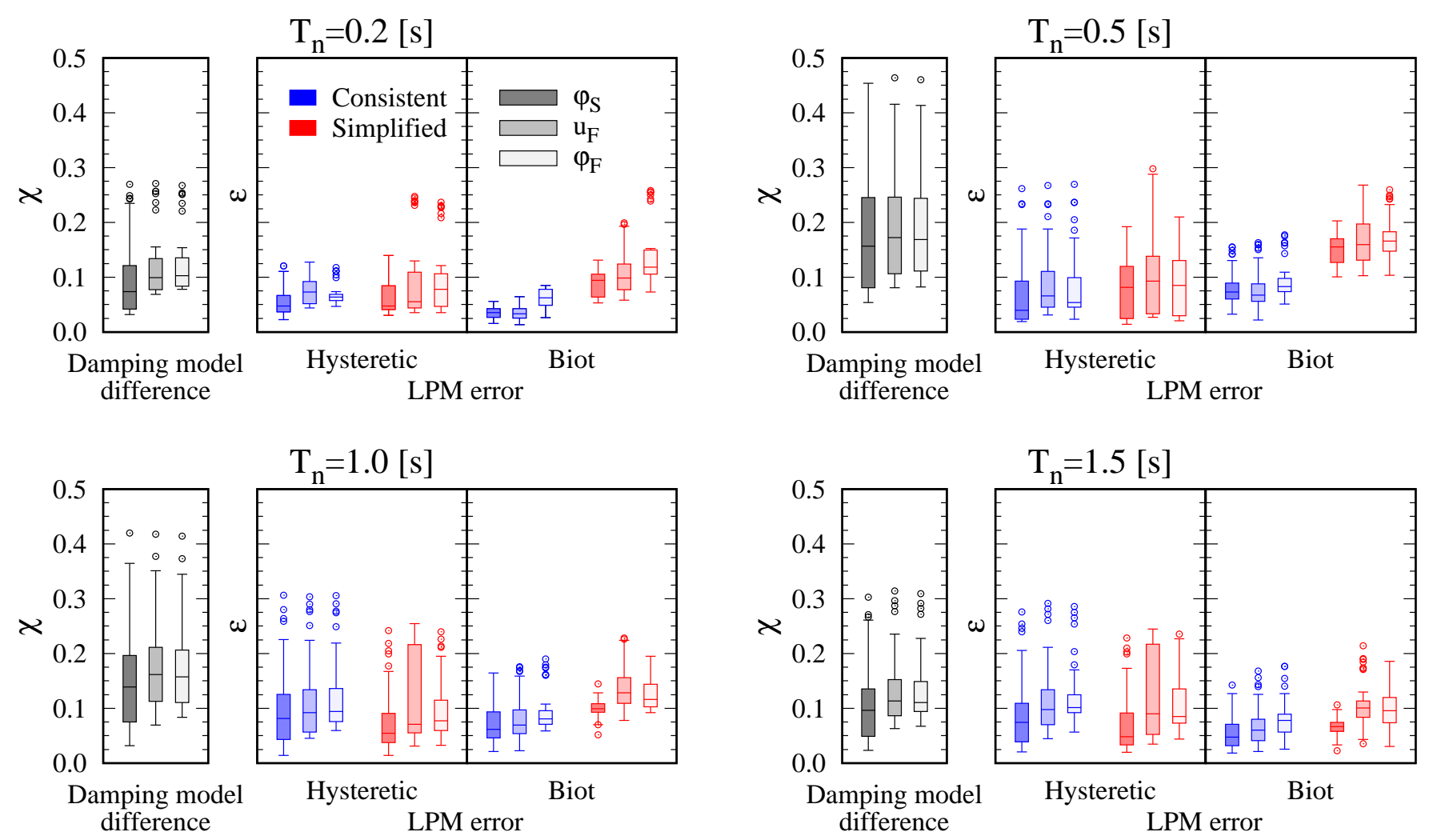

Figure 11: Differences due to the use of hysteretic or Biot's soil damping model, and errors due to the use of LPM approaches. Influence of superstructure fundamental periods (ground type D).

type D. Furthermore, errors are also less scattered around the median value. This is reasonably due to the even simpler trends of the soil-foundation impedances in the frequency range in which the superstructure fundamental frequencies fall, and the lower magnitude of SSI.

\subsubsection{Influence of pile foundation layouts}

Figure 13 presents LPM errors for all configurations in type D grounds, grouped by foundation layout. As well known, by increasing the pile spacing, peaks of the soil-foundation impedances due to group effects move towards lower frequencies. Thus, for increasing pile spacing, the simplified LPM is no longer able to capture the actual frequency behaviour of the soil-foundation impedances in the selected frequency range. This observation is consistent with the significant increase of the overall errors observed for the $3 \times 3$ cases when increasing the pile spacing (of around $100 \%$ from $s=3 \mathrm{~m}$ to $s=7 \mathrm{~m}$ ), independently from the adopted soil damping model. It is also consistent with the fact that, for $s=7 \mathrm{~m}$, the errors committed when using the simplified LPM are significantly larger than those committed when using the consistent approach, for both $2 \times 2$ and $3 \times 3$ configurations. For the $2 \times 2$ pile foundations, an increase of errors with pile spacing is not observed because pile group resonances do not arise in impedance functions in the frequency range $0-6 \mathrm{~Hz}$.

The largest errors arise for the softest soil-foundation configuration $(2 \times 2$ and $s=3 \mathrm{~m})$ when a hysteretic soil damping model is assumed, with errors much larger than those for Biot's option or for other configurations. This is caused by the CB fundamental frequency falling below $0.9 \mathrm{~Hz}$, i.e. in a range where none of the LPM approaches is able to capture the unbounded tendency of the damping function, and where, for the analysed cases, the SSI effects are more relevant. Therefore, differences between reference and approximated impedance functions have more influence on the final results.

Regarding the influence of soil damping model on the accuracies, when the hysteretic damping model is assumed, the results obtained when using the simplified LPM tends to be better than those 

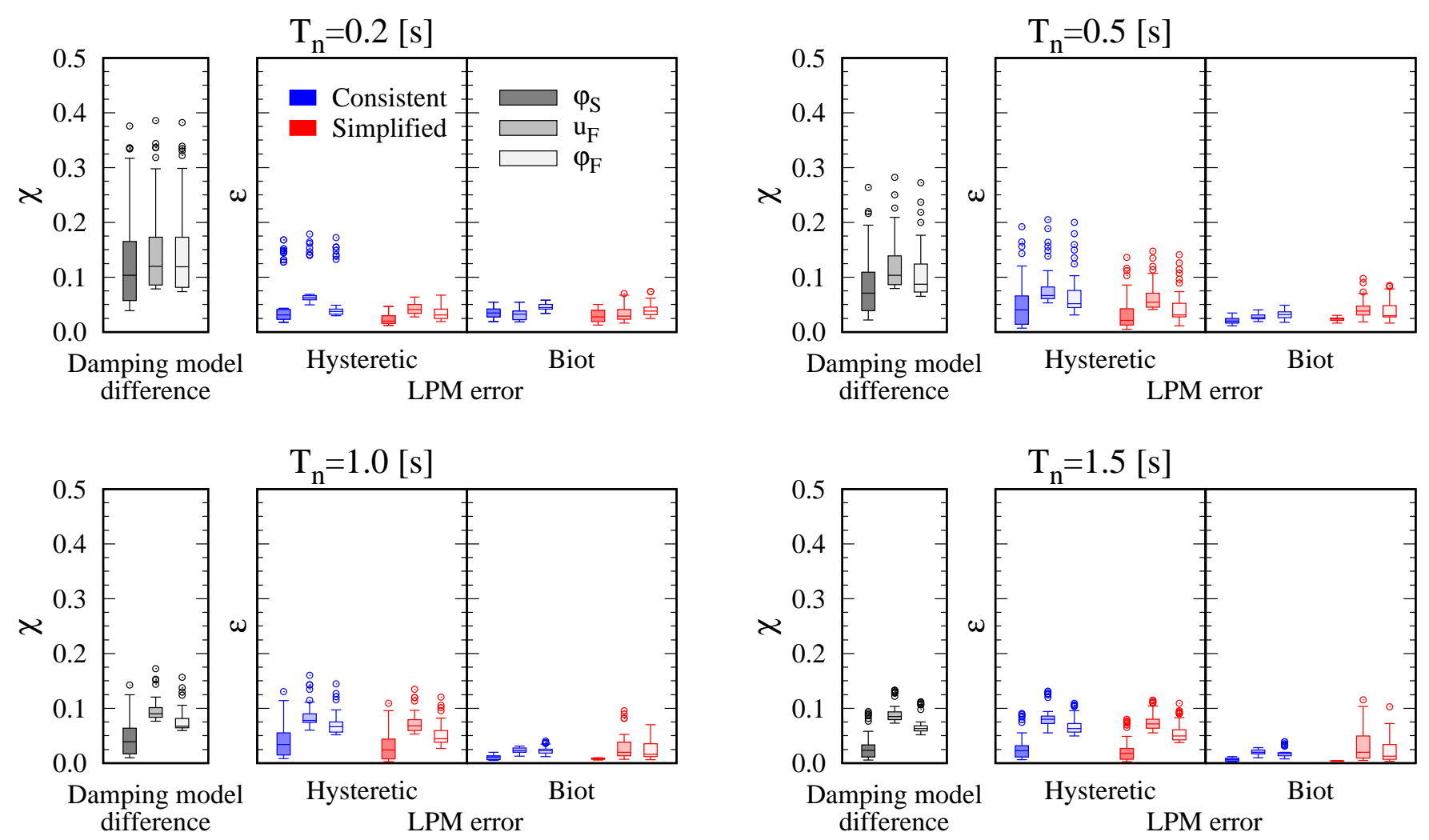

Figure 12: Differences due to the use of hysteretic or Biot's soil damping model, and errors due to the use of LPM approaches. Influence of superstructure fundamental periods (ground type C).

obtained from the consistent option (except for $s=7 \mathrm{~m}$ ). On the contrary, when Biot's soil damping model is assumed, the results obtained when using the consistent LPM tends to be better (except for the $3 \times 3$ and $s=3 \mathrm{~m}$ configuration).

\section{Conclusions}

A wide parametric analysis has been performed in order to elucidate the influence of soil damping model and type of LPM on the computed seismic response of bridge piers on pile groups in cases where soil-structure interaction phenomena are relevant. The study involved 4 different bridge pier superstructures, 6 pile foundation layouts, 2 soil deposits and 2 soil damping models (classical hysteretic and Biot's damping model), with the system subjected to 7 real earthquake signals scaled for each soil type. The response of the system was computed using a substructuring approach in the frequency domain and also in the time domain by approximating the response of the foundation using two alternative lumped parameter models of different complexity. Including also fixed base cases for reference, 2072 different cases in total were studied.

The impedance and kinematic interaction functions (previously computed using a harmonic 3D BEM-FEM code) are dependent on the chosen soil damping model. The most relevant differences appear in the low-frequency part of the damping functions, as the damping coefficient provided by the classical hysteretic model is unbounded at zero frequency, which leads to numerical difficulties for its fitting with lumped parameter models. At higher frequencies, on the contrary, differences grow in the stiffness and the kinematic interaction functions.

Both the consistent and the simplified lumped parameter models lead to approximated impedance functions that are continuous and symmetric with respect to frequency, which make them unable to fit the nonphysicial unbounded tendency of the damping coefficient of the classical hysteretic damping 

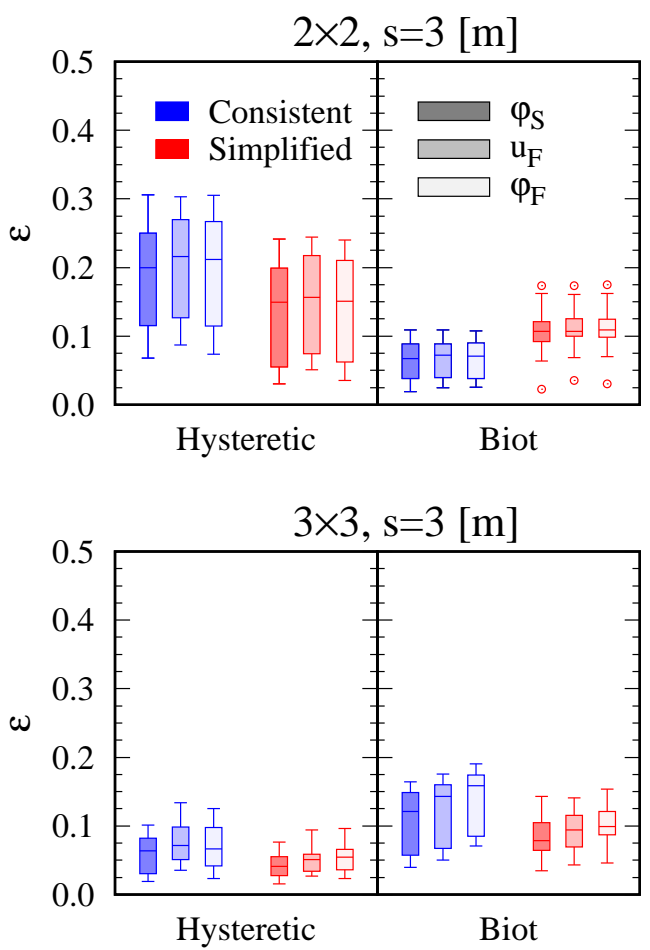

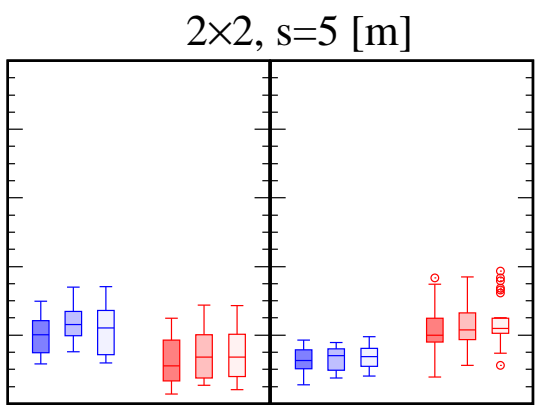

Hysteretic

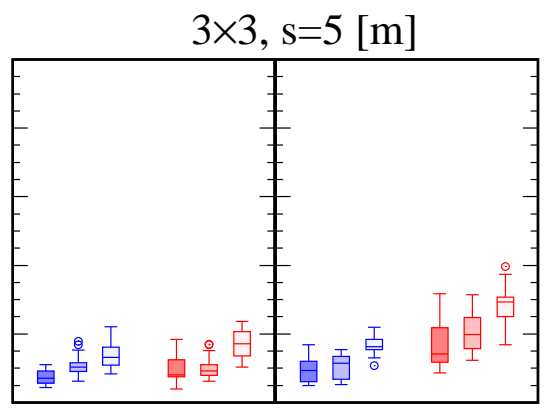

Hysteretic

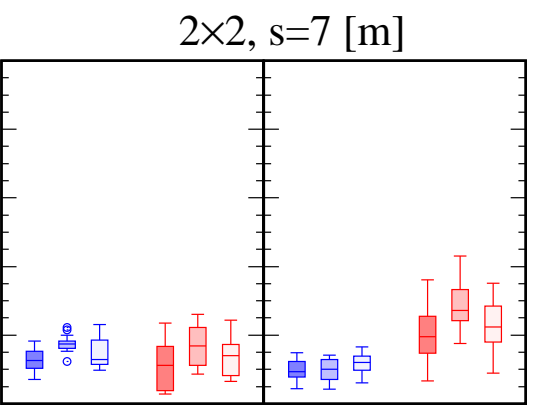

Hysteretic

Biot

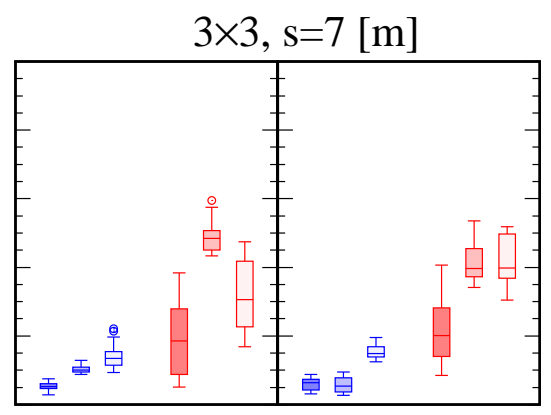

Hysteretic

Biot

Figure 13: Differences due to the use of hysteretic or Biot's soil damping model, and errors due to the use of LPM approaches. Influence of foundation layouts (ground type D.)

model at zero frequency. The consequence is the impossibility of a good matching at very low frequencies when such hysteretic damping model is assumed for the soil, which is of special relevance in bridge piers, characterized by low fundamental frequencies. In any case, from an engineering point of view, both LPMs used in the study provide, in general, sufficiently low errors.

The magnitude of the influence of the soil damping model adopted for the study is, as could not be otherwise, not as large as that of the SSI effects, being the influence at the foundation level more relevant than that at the superstructure. It is also worth noting that larger displacements at the deck are predicted when Biot's model is adopted for the soil instead of the classical hysteretic model (due to the higher damping at low frequencies provided by the latter one). At the same time, the errors induced by approximating the impedance functions through the consistent or the simplified LPM are generally slightly lower than the differences arising from assuming the classical hysteretic or the causal Biot's models, i.e., the use of the simplified or the more accurate consistent LPM has, in general, less influence on the computed responses than the different assumptions that are made in the process of building the structural model. In terms of structural integrity, the assumption of Biot's damping together with the adoption of the consistent lumped parameter model could be adopted as the most conservative option, although the limited magnitude of the differences among approaches can justify the use of the simplest approach in most situations. However, it is worth noting that, in the present manuscript, superstructures are modelled as one degree-of-freedom systems in their fixed base configuration, and are characterized by their fundamental frequency. This is one of the reasons why even the simplified LPM, for which fitting errors can be significant after a given frequency, provides good results in most of the cases studied herein. Therefore, for other types of superstructures, better characterized as multi degree-of-freedom systems, more elaborate lumped parameter models able to represent impedance functions accurately in a wider range of frequencies (such as the consistent approach) would possibly be needed in order to represent correctly the contribution of all vibration modes, so the simplest approaches would probably not be suitable. The non-physical behaviour provided by the classical hysteretic damping model for the soil at zero frequency makes the overall error committed by using the simplified LPM to model the foundation 
response smaller than the one obtained when using the more elaborate consistent approach for medium stiffness soil deposits. On the contrary, when assuming the causal Biot's damping model for the soil, the consistent LPM leads to consistently more accurate results.

The study has been performed assuming a linear elastic behaviour for both the soil-foundation system and the superstructure. Concerning the soil-foundation system, the assumption of linearity is generally adopted dealing with soil structure interaction problems, justified by the capacity design of foundations and the complexity of handling the complex frequency-dependent non-linear nature of soil. As for the superstructures, the assumption of linearity is realistic for low and moderate intensity earthquakes while for high intensity events plastic hinges at the piers base are expected to develop. Nevertheless, in the latter cases, linear approaches may provide an estimate of the displacement demand of non-linear piers, according to the well-known equal energy and equal displacement rules [15]. It is also important to highlight the fact that some of the conclusions can also change when layered grounds are studied, as the appearance of cut-off frequencies will affect the ability of both LPMs, but especially the simplified approach, to catch more intricate impedance functions, and will also affect the influence of the adoption of one soil damping model or the other. These aspects should be tackled in future research.

\section{Acknowledgements}

This study was supported by the Ministerio de Economía, Industria y Competitividad and the Agencia Estatal de Investigación of Spain, and FEDER, through research projects BIA2014-57640$\mathrm{R}$ and BIA2017-88770-R. F. González is recipient of the FPU fellowship FPU14/06936 from the Ministerio de Educación, Cultura y Deporte of Spain, which also provided him with financial support (EST16/00392) for a short-term research stay at Università Politecnica delle Marche.

\section{References}

[1] G Mylonakis, A Nikolaou, and G Gazetas. Soil-pile-bridge seismic interaction: kinematic and inertial effects. Part I: soft soil. Earthquake Engineering 85 Structural Dynamics, 26(3):337-359, 1997.

[2] MTA Chaudhary, M Abé, and Y Fujino. Identification of soil-structure interaction effect in base-isolated bridges from earthquake records. Soil Dynamics and Earthquake Engineering, 21(8):713-725, 2001.

[3] G Mylonakis, S Nikolaou, and G Gazetas. Footings under seismic loading: analysis and design issues with emphasis on bridge foundations. Soil Dynamics and Earthquake Engineering, 26(9):824-853, 2006.

[4] S Carbonari, F Dezi, and G Leoni. Seismic soil-structure interaction in multi-span bridges: application to a railway bridge. Earthquake Engineering \& Structural Dynamics, 40(11):12191239, 2011.

[5] JP Wolf and DR Somaini. Approximate dynamic model of embedded foundation in time domain. Earthquake Engineering \&6 Structural Dynamics, 14(5):683-703, 1986.

[6] M Saitoh. Simple model of frequency-dependent impedance functions in soil-structure interaction using frequency-independent elements. Journal of Engineering Mechanics, 133(10):11011114, 2007. 
[7] N Lesgidis, O-S Kwon, and A Sextos. A time-domain seismic SSI analysis method for inelastic bridge structures through the use of a frequency-dependent lumped parameter model. Earthquake Engineering \& Structural Dynamics, 44(13):2137-2156, 2015.

[8] JP Wolf. Consistent lumped-parameter models for unbounded soil: physical representation. Earthquake Engineering \& Structural Dynamics, 20(1):11-32, 1991.

[9] JP Wolf. Consistent lumped-parameter models for unbounded soil: frequency-independent stiffness, damping and mass matrices. Earthquake Engineering \& Structural Dynamics, 20(1):3341, 1991.

[10] JW Meek and AS Veletsos. Simple models for foundations in lateral and rocking motion. In Proceedings of the 5th World Conference on Earthquake Engineering, 2:2610-2613, 1974.

[11] FCP De Barros and JE Luco. Discrete models for vertical vibrations of surface and embedded foundations. Earthquake Engineering \& Structural Dynamics, 19(2):289-303, 1990.

[12] W-Y Jean, T-W Lin, and J Penzien. System parameters of soil foundations for time domain dynamic analysis. Earthquake Engineering \& Structural Dynamics, 19(4):541-553, 1990.

[13] EN1998-5. Eurocode 8 - Design of structures for earthquake resistance. Part 5: foundations, retaining structures and geotechnical aspects. CEN, 2004.

[14] RED Bishop. The treatment of damping forces in vibration theory. Journal of the Royal Aeronautical Society, 59(539):738-742, 1955.

[15] AK Chopra. Dynamics of structures. Theory and applications to earthquake engineering. Third edition. Prentice-Hall, 2007.

[16] HC Booij and GPJM Thoone. Generalization of Kramers-Kronig transforms and some approximations of relations between viscoelastic quantities. Rheologica Acta, 21(1):15-24, 1982.

[17] MA Biot. Linear thermodynamics and the mechanics of solids. In Proceedings of the 3rd U. S. National Congress of Applied Mechanics, 1:1-18, 1958.

[18] N Makris. Causal hysteretic element. Journal of Engineering Mechanics, 123(11):1209-1214, 1997.

[19] S Carbonari, M Morici, F Dezi, F Gara, and G Leoni. Soil-structure interaction effects in single bridge piers founded on inclined pile groups. Soil Dynamics and Earthquake Engineering, 92:52-67, 2017.

[20] E Kausel, RV Whitman, JP Morray, and F Elsabee. The spring method for embedded foundations. Nuclear Engineering and Design, 48(2):377-392, 1978.

[21] G Gazetas, K Fan, T Tazoh, K Shimizu, M Kavvadas, and N Makris. Seismic pile-groupstructure interaction. Geotecnical special pulication, ASCE, 34:56-93, 1992.

[22] EN1998-1. Eurocode 8 - Design of structures for earthquake resistance. Part 1: general rules, seismic actions and rules for buildings. CEN, 2004.

[23] NN Ambraseys, P Smit, J Douglas, B Margaris, R Sigbjörnsson, S Ólafsson, P Suhadolc, and $\mathrm{G}$ Costa. Internet site for European strong-motion data. Bollettino di Geofisica Teorica ed Applicata, 45(3):113-129, 2004. 
[24] L Luzi, F Pacor, and R Puglia. Italian Accelerometric Archive v 2.2. Istituto Nazionale di Geofisica e Vulcanologia, Dipartimento della Protezione Civile Nazionale, 2017.

[25] N Luco and P Bazzurro. Effects of earthquake record scaling on nonlinear structural response. Report on PEER-LL Program Task 1G00 Addendum (Sub-Task 1 of 3), 2005.

[26] JW Baker. Measuring bias in structural response caused by ground motion scaling. In Proceedings of the 8th Pacific Conference on Earthquake Engineering. Paper Number 056, 2007.

[27] EI Katsanos, AG Sextos, and GD Manolis. Selection of earthquake ground motion records: A state-of-the-art review from a structural engineering perspective. Soil Dynamics and Earthquake Engineering, 30(4):157-169, 2010.

[28] JP Wolf. Soil-structure interaction analysis in time domain. Prentice-Hall, 1988.

[29] LA Padrón, JJ Aznárez, and O Maeso. BEM-FEM coupling model for the dynamic analysis of piles and pile groups. Engineering Analysis with Boundary Elements, 31(6):473-484, 2007.

[30] AL Kimball and DE Lovell. Internal friction in solids. Physical Review, 30(6):948-959, 1927.

[31] GB Muravskii. On frequency independent damping. Journal of Sound and Vibration, 274(35):653-668, 2004.

[32] GB Muravskii. Linear models with nearly frequency independent complex stiffness leading to causal behaviour in time domain. Earthquake Engineering 8 Structural Dynamics, 36(1):13-33, 2007.

[33] L Andersen. Linear elastodynamic analysis. Department of Civil Engineering, Aalborg University, 2006.

[34] L Andersen. Assessment of lumped-parameter models for rigid footings. Computers \& Structures, 88(23-24):1333-1347, 2010.

[35] F González, LA Padrón, JJ Aznárez, and O Maeso. Implementation of the consistent lumpedparameter model for the computation of the seismic response of nonlinear piled structures. In Proceedings of the 10th International Conference on Structural Dynamics, Procedia Engineering, 199:2360-2365, 2017.

[36] S Carbonari, M Morici, F Dezi, and G Leoni. A lumped parameter model for time-domain inertial soil-structure interaction analysis of structures on pile foundations. Earthquake Engineering E Structural Dynamics, 47(11):2147-2171, 2018.

[37] A Paronesso and JP Wolf. Global lumped-parameter model with physical representation for unbounded medium. Earthquake Engineering $\& 5$ Structural Dynamics, 24(5):637-654, 1995. 